$5-15-2020$

\title{
A New Exponential Approach for Reducing the Mean Squared Errors of the Estimators of Population Mean Using Conventional and Non-Conventional Location Parameters
}

\author{
Housila P. Singh \\ Vikram University, Ujjain, India, hpsujn@gmail.com \\ Anita Yadav \\ Vikram University, Ujjain, India, yadavd.anita@gmail.com
}

Follow this and additional works at: https://digitalcommons.wayne.edu/jmasm

Part of the Applied Statistics Commons, Social and Behavioral Sciences Commons, and the Statistical Theory Commons

\section{Recommended Citation}

Singh, Housila P. and Yadav, Anita (2020) "A New Exponential Approach for Reducing the Mean Squared Errors of the Estimators of Population Mean Using Conventional and Non-Conventional Location Parameters," Journal of Modern Applied Statistical Methods: Vol. 18 : Iss. 1 , Article 26. DOI: $10.22237 / \mathrm{jmasm} / 1568246400$ Available at: https://digitalcommons.wayne.edu/jmasm/vol18/iss1/26

This Regular Article is brought to you for free and open access by the Open Access Journals at DigitalCommons@WayneState. It has been accepted for inclusion in Journal of Modern Applied Statistical Methods by an authorized editor of DigitalCommons@WayneState. 


\section{A New Exponential Approach for Reducing the Mean Squared Errors of the Estimators of Population Mean Using Conventional and Non-Conventional Location Parameteres}

\author{
Housila P. Singh \\ Vikram University \\ Ujjain, India
}

\author{
Anita Yadav \\ Vikram University \\ Ujjain, India
}

Classes of ratio-type estimators $t$ (say) and ratio-type exponential estimators $t_{e}$ (say) of the population mean are proposed, and their biases and mean squared errors under large sample approximation are presented. It is the class of ratio-type exponential estimators $t_{e}$ provides estimators more efficient than the ratio-type estimators.

Keywords: study variable, auxiliary variable, bias, mean squared error.

\section{Introduction}

The use of auxiliary information at the estimation stage of a survey improves the precision of the estimate(s) of the parameter(s) under investigation. The problem of estimating the population mean or total using population mean of an auxiliary variable has been extensively discussed. Out of many ratio, product and regression methods of estimation are good examples in this context. The ratio method of estimation is most effective for estimating population mean of the study variable when there is a linear relationship between study variable and auxiliary variable and they have the positive (high) correlation. However, if the correlation between study variable and auxiliary variable is negative (high) the product method of estimation can be employed.

Let $U=\left(U_{1}, U_{2}, \ldots, U_{N}\right)$ be the finite population of size $N$ and the variables under study and auxiliary be denoted by y and $\mathrm{x}$ respectively. Let $(\hat{\bar{Y}}, \hat{\bar{X}})$ be the population means of $(y, x)$ respectively. It is desired to estimate the population mean $\bar{Y}$ 
using information on population parameters such as mean $(\bar{X})$, coefficient of variation $\left(C_{x}\right)$, coefficient of skewness $\left(\beta_{1}(x)\right)$, kurtosis $\left(\beta_{2}(x)\right)$, deciles, quartiles, median, midrange (MR), Walsh average (i.e. Hodges-Lehman estimator) (HL) (and tri mean (TM) etc, associated with auxiliary variable $x$ and the correlation coefficient $\rho$ between y and $x$. In this context, the reader is referred to Searls (1964), Das and Tripathi (1980), Sisodia and Dwivedi (1981), Upadhyaya and Singh (1999), Singh and Tailor (2003), Singh et al (2004), Kadilar and Cingi (2004, 2006), Yan and Tian (2010), Subramani and Kumarapandian (2012a,2012b,2012c), Jeelani et al (2013), Ekpenyoung and Enang (2015), Subramani et al (2015) and Abid et al (2016a,b,c).

Define:

$N$ : Population size.

$n$ : Sample size.

$f=\frac{n}{N}:$ Sampling fraction.

$\theta=\frac{(1-f)}{n}$.

$S_{y}^{2}=(N-1)^{-1} \sum_{i=1}^{N}\left(y_{i}-\bar{Y}\right)^{2}$ : Population Variance of the study variable $\mathrm{y}$.

$S_{x}^{2}=(N-1)^{-1} \sum_{i=1}^{N}\left(x_{i}-\bar{X}\right)^{2}$ : Population variance of the auxiliary variable $x$.

$C_{y}=S_{y} / \bar{Y}:$ Coefficient of variation of the study variable y.

$C_{x}=S_{x} / \bar{X}$ : Coefficient of variation of the auxiliary variable $x$.

$S_{y}=(N-1)^{-1} \sum_{i=1}^{N}\left(x_{i}-\bar{X}\right)\left(y_{i}-\bar{Y}\right)$ : Covariance between $y$ and $x$.

$\rho=S_{y} /\left(S_{x} S_{y}\right):$ Correlation coefficient between $x$ and $y$.

$C=\rho C_{y} / C_{x}$,

$M_{d}$ : Population median of $\mathrm{x}$.

$Q_{i}: i^{\text {th }}$ population quartile $(\mathrm{i}=1,2,3)$.

$T_{m}: \frac{\left(Q_{1}+Q_{2}+Q_{3}\right)}{2}:$ Tri mean. 


\section{REDUCING THE MSE OF POPULATION ESTIMATORS}

$H_{l}=\operatorname{median}\left(\left(X_{j}+X_{k}\right) / 2,1 \leq j \leq k \leq N\right)$ Hodges-Lehmann estimator.

$X_{(1)}$ : Lowest order statistic in a population of size N,

$X_{(N)}$ : Highest order statistic in a population of size $\mathrm{N}$,

$M_{r}=\frac{X_{(1)}+X_{(N)}}{2}:$ Mid range,

$Q_{r}=\left(Q_{3}-Q_{1}\right):$ Inter-quartile range,

$Q_{d}=\frac{Q_{3}-Q_{1}}{2}:$ Semi-quartile range,

$\beta_{1}(x)=\frac{N i \sum_{i=1}^{N}\left(x_{i}-\bar{X}\right)^{3}}{(N-1)(N-2) S_{x}^{3}}$ : Coefficient of skewness of the auxiliary variable $\mathrm{x}$,

$\beta_{2}(x)=\left[\frac{N(N+1) \sum_{i=1}^{N}\left(x_{i}-\bar{X}\right)^{4}}{(N-1)(N-2)(N-3) S_{x}^{4}}-\frac{3(N-1)^{2}}{(N-2)(N-3)}\right]:$ Coefficient of kurtosis of the auxiliary variable $\mathrm{x}$,

$\Delta=\left[\beta_{2}(x)-\beta_{1}^{2}(x)+\frac{2(N-1)^{2}}{(N-2)(N-3)}\right]$,

$Q_{a}=\frac{Q_{3}+Q_{1}}{2}:$ uartile average,

$R=\frac{\bar{Y}}{\bar{X}}:$ Population ratio of means,

$G=\frac{4}{N-1} \sum_{i=1}^{N} \frac{(2 i-N-1)}{2 N} X_{(i)}$ : Gini's Mean Difference,

$D=\frac{2 \sqrt{\pi}}{N(N-1)} \sum_{i=1}^{N}\left(i-\frac{N+1}{2}\right) X_{(i)}$ : Downton's method,

$S_{p w}=\frac{\sqrt{\pi}}{N^{2}} \sum_{i=1}^{N}(2 i-N-1) X_{(i)}$ : Probability Weighted Moments,

$\mu_{r}(x)=(1 / N) \sum_{i=1}^{N}\left(x_{i}-\bar{X}\right)^{r}$ : r being non-negative integer. 
We are interested in estimating the population mean $\bar{Y}$ of the study variable $y$ (taking value $\mathrm{y}_{\mathrm{i}}$ for $\mathrm{i}=1,2, \ldots, N$ ) from a simple random sample size $\mathrm{n}$ drawn without replacement from the population $U$. We use the notation $\bar{y}$ and $\bar{x}$ for the sample means, which are unbiased estimators of the population mean $\bar{Y}$ and $\bar{X}$, respectively. We also denote:

$$
\begin{aligned}
& s_{x y}=(n-1)^{-1} \sum_{i=1}^{n}\left(x_{i}-\bar{x}\right)\left(y_{i}-\bar{y}\right): \text { Sample covariance between } y \text { and } x . \\
& s_{x}^{2}=(n-1)^{-1} \sum_{i=1}^{n}\left(x_{i}-\bar{x}\right)^{2}: \text { Sample variance of } x . \\
& \hat{\beta}=\frac{s_{x y}}{s_{x}^{2}} \text { : Sample regression coefficient estimate of the population regression }
\end{aligned}
$$
coefficient $\beta=\frac{S_{x y}}{S_{x}^{2}}$ of y on x.

$$
\begin{aligned}
& \hat{R}=\frac{\bar{y}}{\bar{x}}: \text { Ratio of sample means. } \\
& \hat{\bar{Y}}=[\bar{y}+\hat{\beta}(\bar{X}-\bar{x})]: \text { Regression estimator of the population mean } \bar{Y} .
\end{aligned}
$$

\section{Existing Modified Ratio Estimators and The Suggested Class of Ratio Estimators}

The usual unbiased estimator for population mean $\bar{Y}$ is defined by

$$
t_{0}=\bar{y}
$$

whose MSE is given by

$$
\operatorname{MSE}(\bar{y})=\frac{(1-f)}{n} S_{y}^{2} .
$$

The classical ratio estimator for the population mean $\bar{Y}$ in presence of known population mean $\bar{X}$ of the auxiliary variable $\mathrm{x}$ is defined by

$$
\bar{y}_{R}=\bar{y} \frac{\bar{X}}{\bar{x}} \bar{x} \neq 0 .
$$

To the first degree of approximation, the bias and MSE of the ratio estimator $\bar{y}_{R}$ are respectively given by

$$
B\left(\bar{y}_{R}\right)=\frac{(1-f)}{n} \cdot \frac{1}{\bar{X}}\left(R S_{x}^{2}-\rho S_{x} S_{y}\right)
$$




\section{REDUCING THE MSE OF POPULATION ESTIMATORS}

and

$$
\operatorname{MSE}\left(\bar{y}_{R}\right)=\frac{(1-f)}{n}\left(S_{y}^{2}+R^{2} S_{x}^{2}-2 R \rho S_{y} S_{x}\right)
$$

In Table 1, the modified versions of the ratio estimator reported by Kadilar and Cingi (2004) are given, Kadilar and Cingi (2006) -type estimator, Yan and Tian (2010), Subramani and Kumarapandiyan (2012a, 2012b, 2012c, 2012d), Jeelani et al. (2013) and Abid et al. (2016) along with their biases and mean squared errors (MSEs) to the first degree of approximation, as reported in Abid et al. (2016).

Note the estimators $t_{j}(j=1$ to 65$)$ members of the following class of estimators of the population mean $\bar{Y}$ defined by

$$
t=\hat{\bar{Y}}\left(\frac{a \bar{X}+b}{a \bar{x}+b}\right)=[\bar{y}+\hat{\beta}(\bar{X}-\bar{x})]\left(\frac{a \bar{X}+b}{a \bar{x}+b}\right),
$$

where $\hat{\beta}$ is the sample estimate of the population regression coefficient $\beta$ of $\mathrm{y}$ on $\mathrm{x}$, $\mathrm{a}(\neq 0)$ and $\mathrm{b}$ are real numbers (constants) or the functions of population parameters such as population total $X(=N \bar{X})$, population standard deviation $S_{x}$, variance $S_{x}^{2}$, coefficient of variation $C_{x}$,

Coefficient of skewness $\beta_{1}(x)$ and kurtosis $\beta_{2}(x)$, correlation coefficient $\rho$, $\Delta$, quartiles, deciles, median, mode, midrange, Trimean and Hodgs-Lehmann (HL) estimator etc.

Some unknown members of the suggested class of ratio-type estimators $t$ are given in Table 2

To obtain the bias and mean squared error of the proposed class of estimators ' $t$ ' we write

$$
\bar{y}=\bar{Y}\left(1+e_{0}\right), \bar{x}=\bar{X}\left(1+e_{1}\right), s_{x y}=S_{x y}\left(1+e_{2}\right), s_{x}^{2}=S_{x}^{2}\left(1+e_{3}\right)
$$

such that

$$
E\left(e_{i}\right)=0 \text { for all } i=1,2,3 \text {; }
$$

and

$$
\begin{gathered}
E\left(e_{0}^{2}\right)=\frac{(1-f)}{n} C_{y}^{2}, E\left(e_{1}^{2}\right)=\frac{(1-f)}{n} C_{x}^{2}, E\left(e_{0} e_{1}\right)=\frac{(1-f)}{n} C C_{x}^{2}, \\
E\left(e_{1} e_{2}\right)=\frac{N(N-n)}{(N-1)(N-2)} \frac{\mu_{21}}{n} \frac{1}{\bar{X} S_{x y}}=\frac{(N-n)}{n(N-2)} \frac{\mu_{21}}{\bar{X} \mu_{11}},
\end{gathered}
$$




\section{HOUSILA P. SINGH \& ANITA YADAV}

Table 1. Known modified ratio estimators for the population mean $\bar{Y}$.

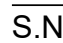

S.No.

Estimator MSE of (.)

Population Ratio

1. $t_{1}=\frac{\hat{\bar{Y}}}{\bar{x}} \cdot \bar{X}$

$$
\theta\left[R_{1}^{2} S_{x}^{2}+S_{y}^{2}\left(1-\rho^{2}\right)\right] \quad R_{1}=\frac{\bar{Y}}{\bar{X}}=R
$$

Kadilar and Cingi (2004)

2. $t_{2}=\frac{\hat{\bar{Y}}}{\left(\bar{x}+C_{x}\right)}\left(\bar{X}+C_{x}\right)$

$$
\theta\left[R_{2}^{2} S_{x}^{2}+S_{y}^{2}\left(1-\rho^{2}\right)\right] \quad R_{2}=\frac{\bar{Y}}{\left(\bar{X}+C_{x}\right)}
$$

Kadilar and Cingi (2004)

3. $t_{3}=\frac{\hat{\bar{Y}}}{\left(\bar{x}+\beta_{2}(x)\right)}\left(\bar{X}+\beta_{2}(x)\right)$

$$
\theta\left[R_{3}^{2} S_{x}^{2}+S_{y}^{2}\left(1-\rho^{2}\right)\right] \quad R_{3}=\frac{\bar{Y}}{\bar{X}+\beta_{2}(x)}
$$

Kadilar and Cingi (2004)

4. $\quad t_{4}=\frac{\hat{\bar{Y}}}{\left(\bar{x} \beta_{2}(x)+C_{x}\right)}\left(\bar{X} \beta_{2}(x)+C_{x}\right) \quad \theta\left[R_{4}^{2} S_{x}^{2}+S_{y}^{2}\left(1-\rho^{2}\right)\right] \quad R_{4}=\frac{\bar{Y} \beta_{2}(x)}{\left(\bar{X} \beta_{2}(x)+C_{x}\right)}$

Kadilar and Cingi (2004)

5. $\quad t_{5}=\frac{\hat{\bar{Y}}}{\left(\bar{x} C_{x}+\beta_{2}(x)\right)}\left(\bar{X} C_{x}+\beta_{2}(x)\right) \quad \theta\left[R_{5}^{2} S_{x}^{2}+S_{y}^{2}\left(1-\rho^{2}\right)\right] \quad R_{5}=\frac{\bar{Y} C_{x}}{\left(\bar{X} C_{x}+\beta_{2}(x)\right)}$

Kadilar and Cingi (2004)

6. $\quad t_{6}=\frac{\hat{\bar{Y}}}{(\bar{x}+\rho)}(\bar{X}+\rho)$

$$
\theta\left[R_{6}^{2} S_{x}^{2}+S_{y}^{2}\left(1-\rho^{2}\right)\right] \quad R_{6}=\frac{\bar{Y}}{\bar{X}+\rho}
$$

Kadilar and Cingi (2006) -type

7. $\quad t_{7}=\frac{\hat{\bar{Y}}}{\left(\bar{x} C_{x}+\rho\right)}\left(\bar{X} C_{x}+\rho\right)$

$$
\theta\left[R_{7}^{2} S_{x}^{2}+S_{y}^{2}\left(1-\rho^{2}\right)\right] \quad R_{7}=\frac{\bar{Y} C_{x}}{\left(\bar{X} C_{x}+\rho\right)}
$$

Kadilar and Cingi (2006) -type

8. $\quad t_{8}=\frac{\hat{\bar{Y}}}{\left(\bar{x} \rho+C_{x}\right)}\left(\bar{X} \rho+C_{x}\right) \quad \theta\left[R_{8}^{2} S_{x}^{2}+S_{y}^{2}\left(1-\rho^{2}\right)\right] \quad R_{8}=\frac{\bar{Y} \rho}{\left(\bar{X} \rho+C_{x}\right)}$

Kadilar and Cingi (2006) -type

9. $\quad t_{9}=\frac{\hat{\bar{Y}}}{\left(\bar{x} \beta_{2}(x)+\rho\right)}\left(\bar{X} \beta_{2}(x)+\rho\right)$

Kadilar and Cingi (2006) -type

10. $\quad t_{10}=\frac{\hat{\bar{Y}}}{\left(\bar{x} \rho+\beta_{2}(x)\right)}\left(\bar{X} \rho+\beta_{2}(x)\right)$

Kadilar and Cingi (2006) -type

11. $\quad t_{11}=\frac{\hat{\bar{Y}}}{\left(\bar{x}+\beta_{1}(x)\right)}\left(\bar{X}+\beta_{1}(x)\right)$

$$
\theta\left[R_{9}^{2} S_{x}^{2}+S_{y}^{2}\left(1-\rho^{2}\right)\right] \quad R_{9}=\frac{\bar{Y} \beta_{2}(x)}{\left(\bar{X} \beta_{2}(x)+\rho\right)}
$$

Yan and Tian (2010) 


\section{REDUCING THE MSE OF POPULATION ESTIMATORS}

Table 1. continued

S.No. Estimator
MSE of (.) Population Ratio

12. $\quad t_{12}=\frac{\hat{\bar{Y}}}{\left(\bar{x} \beta_{1}(x)+\beta_{2}(x)\right)}\left(\bar{X} \beta_{1}(x)+\beta_{2}(x)\right) \quad \theta\left[R_{12}^{2} S_{x}^{2}+S_{y}^{2}\left(1-\rho^{2}\right)\right] \quad R_{12}=\frac{\bar{Y} \beta_{1}(x)}{\left(\bar{X} \beta_{1}(x)+\beta_{2}(x)\right)}$ Yan and Tian (2010)

13. $\quad t_{13}=\frac{\hat{\bar{Y}}}{\left(\bar{x}+M_{d}\right)}\left(\bar{X}+M_{d}\right)$

Subramani and Kumarapandian (2012a)

14. $\quad t_{14}=\frac{\hat{\bar{Y}}}{\left(C_{x} \bar{x}+M_{d}\right)}\left(\bar{X} C_{x}+M_{d}\right)$

Subramani and Kumarapandiyan (2012a)

15. $\quad t_{15}=\frac{\hat{\bar{Y}}}{\left(\beta_{1}(x) \bar{x}+M_{d}\right)}\left(\bar{X} \beta_{1}(x)+M_{d}\right)$

Subramani and Kumarapandiyan (2012b)

$$
\theta\left[R_{13}^{2} S_{x}^{2}+S_{y}^{2}\left(1-\rho^{2}\right)\right] \quad R_{13}=\frac{\bar{Y}}{\left(\bar{X}+M_{d}\right)}
$$

$t_{16}=\frac{\hat{\bar{Y}}}{\left(\beta_{2}(x) \bar{x}+M_{d}\right)}\left(\bar{X} \beta_{2}(x)+M_{d}\right)$

Subramani and Kumarapandiyan

$$
\theta\left[R_{14}^{2} S_{x}^{2}+S_{y}^{2}\left(1-\rho^{2}\right)\right] \quad R_{14}=\frac{\bar{Y} C_{x}}{\left(\bar{X} C_{x}+M_{d}\right)}
$$
(2012c)

17. $\quad t_{17}=\frac{\hat{\bar{Y}}}{\left(\bar{x}+D_{1}\right)}\left(\bar{X}+D_{1}\right)$

Subramani and Kumarapandiyan (2012d)

$$
\theta\left[R_{15}^{2} S_{x}^{2}+S_{y}^{2}\left(1-\rho^{2}\right)\right] \quad R_{15}=\frac{\beta_{1}(x) \bar{Y}}{\left(\bar{X} \beta_{1}(x)+M_{d}\right)}
$$

16. $t_{18}=\frac{\hat{\bar{Y}}}{\left(\bar{x}+D_{2}\right)}\left(\bar{X}+D_{2}\right)$

Subramani and Kumarapandiyan (2012d)

$$
\theta\left[R_{16}^{2} S_{x}^{2}+S_{y}^{2}\left(1-\rho^{2}\right)\right] \quad R_{16}=\frac{\beta_{2}(x) \bar{Y}}{\left(\bar{X} \beta_{2}(x)+M_{d}\right)}
$$

$t_{19}=\frac{\hat{\bar{Y}}}{\left(\bar{x}+D_{3}\right)}\left(\bar{X}+D_{3}\right)$

Subramani and Kumarapandiyan (2012d)

$$
t_{20}=\frac{\hat{\bar{Y}}}{\left(\bar{x}+D_{4}\right)}\left(\bar{X}+D_{4}\right) \quad \theta\left[R_{20}^{2} S_{x}^{2}+S_{y}^{2}\left(1-\rho^{2}\right)\right] \quad R_{20}=\frac{\bar{Y}}{\left(\bar{X}+D_{4}\right)}
$$

Subramani and Kumarapandiyan (2012d)

$$
\theta\left[R_{19}^{2} S_{x}^{2}+S_{y}^{2}\left(1-\rho^{2}\right)\right] \quad R_{19}=\frac{\bar{Y}}{\left(\bar{X}+D_{3}\right)}
$$

20.

21. $t_{21}=\frac{\hat{\bar{Y}}}{\left(\bar{x}+D_{5}\right)}\left(\bar{X}+D_{5}\right)$

Subramani and Kumarapandiyan(2012d)

$$
\begin{aligned}
& \text { 22. } t_{22}=\frac{\hat{\bar{Y}}}{\left(\bar{x}+D_{6}\right)}\left(\bar{X}+D_{6}\right) \quad \theta\left[R_{22}^{2} S_{x}^{2}+S_{y}^{2}\left(1-\rho^{2}\right)\right] \quad R_{22}=\frac{\bar{Y}}{\left(\bar{X}+D_{6}\right)} \\
& \text { Subramani and Kumarapandiyan (2012d) }
\end{aligned}
$$

$$
\theta\left[R_{21}^{2} S_{x}^{2}+S_{y}^{2}\left(1-\rho^{2}\right)\right] \quad R_{21}=\frac{\bar{Y}}{\left(\bar{X}+D_{5}\right)}
$$




\section{HOUSILA P. SINGH \& ANITA YADAV}

Table 1. continued

S.No. Estimator
MSE of (.)

Population Ratio

23. $\quad t_{23}=\frac{\hat{\bar{Y}}}{(\bar{x}+D 7)}\left(\bar{X}+D_{7}\right)$

Subramani and Kumarapandiyan (2012d)

24. $t_{24}=\frac{\hat{\bar{Y}}}{\left(\bar{x}+D_{8}\right)}\left(\bar{X}+D_{8}\right)$

Subramani and Kumarapandiyan (2012d)

25. $t_{25}=\frac{\hat{\bar{Y}}}{\left(\bar{x}+D_{9}\right)}\left(\bar{X}+D_{9}\right)$

Subramani and Kumarapandiyan (2012d)

$$
\theta\left[R_{23}^{2} S_{x}^{2}+S_{y}^{2}\left(1-\rho^{2}\right)\right] \quad R_{23}=\frac{\bar{Y}}{\left(\bar{X}+D_{7}\right)}
$$

$$
t_{26}=\frac{\hat{\bar{Y}}}{\left(\bar{x}+D_{10}\right)}\left(\bar{X}+D_{10}\right)
$$

Subramani and Kumarapandiyan (2012d)

$$
\theta\left[R_{24}^{2} S_{x}^{2}+S_{y}^{2}\left(1-\rho^{2}\right)\right] \quad R_{24}=\frac{\bar{Y}}{\left(\bar{X}+D_{8}\right)}
$$

$t_{27}=\frac{\hat{\bar{Y}}}{\left(\bar{x} \beta_{1}(x)+Q_{d}\right)}\left(\bar{X} \beta_{1}(x)+Q_{d}\right)$

$$
\theta\left[R_{27}^{2} S_{x}^{2}+S_{y}^{2}\left(1-\rho^{2}\right)\right] \quad R_{27}=\frac{\bar{Y} \beta_{1}(x)}{\left(\bar{X} \beta_{1}(x)+Q_{d}\right)}
$$

Jeelani et al (2013)

28. $\quad t_{28}=\hat{\bar{Y}} \frac{\left(\rho \bar{X}+M_{d}\right)}{\left(\rho \bar{x}+M_{d}\right)}$

$$
\theta\left[R_{28}^{2} S_{x}^{2}+S_{y}^{2}\left(1-\rho^{2}\right)\right] \quad R_{28}=\frac{\rho \bar{Y}}{\left(\rho \bar{X}+M_{d}\right)}
$$

Subramani and Kumarapandiyan (2014)

29. $\quad t_{29}=\frac{\hat{\bar{Y}}}{\left(\beta_{2}(x) \bar{x}+Q_{1}\right)}\left(\bar{X} \beta_{2}(x)+Q_{1}\right)$

$$
\theta\left[R_{29}^{2} S_{x}^{2}+S_{y}^{2}\left(1-\rho^{2}\right)\right] \quad R_{29}=\frac{\beta_{2}(x) \bar{Y}}{\left(\beta_{2}(x) \bar{X}+Q_{1}\right)}
$$

Subramani et al (2014)

30. $\quad t_{30}=\frac{\hat{\bar{Y}}}{\left(\bar{x} \beta_{2}(x)+Q_{3}\right)}\left(\bar{X} \beta_{2}(x)+Q_{3}\right)$

$$
\theta\left[R_{30}^{2} S_{x}^{2}+S_{y}^{2}\left(1-\rho^{2}\right)\right] \quad R_{30}=\frac{\beta_{2}(x) \bar{Y}}{\left(\beta_{2}(x) \bar{X}+Q_{3}\right)}
$$

Subramani et al (2014)

31. $\quad t_{31}=\frac{\hat{\bar{Y}}}{\left(\bar{x} \beta_{2}(x)+Q_{r}\right)}\left(\bar{X} \beta_{2}(x)+Q_{r}\right)$

$$
\theta\left[R_{31}^{2} S_{x}^{2}+S_{y}^{2}\left(1-\rho^{2}\right)\right] \quad R_{31}=\frac{\beta_{2}(x) \bar{Y}}{\left(\beta_{2}(x) \bar{X}+Q_{r}\right)}
$$

Subramani et al (2014)

32. $t_{32}=\frac{\hat{\bar{Y}}}{\left(\bar{x} \beta_{2}(x)+Q_{d}\right)}\left(\bar{X} \beta_{2}(x)+Q_{d}\right)$

$$
\theta\left[R_{32}^{2} S_{x}^{2}+S_{y}^{2}\left(1-\rho^{2}\right)\right] \quad R_{32}=\frac{\beta_{2}(x) \bar{Y}}{\left(\beta_{2}(x) \bar{X}+Q_{d}\right)}
$$

Subramani et al (2014)

33. $\quad t_{33}=\frac{\hat{\bar{Y}}}{\left(\bar{x} \beta_{2}(x)+Q_{a}\right)}\left(\bar{X} \beta_{2}(x)+Q_{a}\right) \quad \theta\left[R_{33}^{2} S_{x}^{2}+S_{y}^{2}\left(1-\rho^{2}\right)\right] \quad R_{33}=\frac{\beta_{2}(x) \bar{Y}}{\left(\beta_{2}(x) \bar{X}+Q_{a}\right)}$

Subramani et al (2014) 


\section{REDUCING THE MSE OF POPULATION ESTIMATORS}

Table 1. continued

S.No. Estimator
MSE of (.)

34. $\quad t_{34}=\frac{\hat{\bar{Y}}}{\left(\bar{x} \beta_{1}(x)+Q_{1}\right)}\left(\bar{X} \beta_{1}(x)+Q_{1}\right)$

$$
\theta\left[R_{34}^{2} S_{x}^{2}+S_{y}^{2}\left(1-\rho^{2}\right)\right] \quad R_{34}=\frac{\beta_{1}(x) \bar{Y}}{\left(\beta_{1}(x) \bar{X}+Q_{1}\right)}
$$

Subramani et al (2014)

35. $\quad t_{35}=\frac{\hat{\bar{Y}}}{\left(\bar{x} \beta_{1}(x)+Q_{3}\right)}\left(\bar{X} \beta_{1}(x)+Q_{3}\right)$

$$
\theta\left[R_{35}^{2} S_{x}^{2}+S_{y}^{2}\left(1-\rho^{2}\right)\right] \quad R_{35}=\frac{\beta_{1}(x) \bar{Y}}{\left(\beta_{1}(x) \bar{X}+Q_{3}\right)}
$$

Subramani et al (2014)

36. $\quad t_{36}=\frac{\hat{\bar{Y}}}{\left(\bar{x} \beta_{1}(x)+Q_{r}\right)}\left(\bar{X} \beta_{1}(x)+Q_{r}\right) \quad \theta\left[R_{36}^{2} S_{x}^{2}+S_{y}^{2}\left(1-\rho^{2}\right)\right] \quad R_{36}=\frac{\beta_{1}(x) \bar{Y}}{\left(\beta_{1}(x) \bar{X}+Q_{r}\right)}$

Subramani et al (2014)

37. $\quad t_{37}=\frac{\hat{\bar{Y}}}{\left(\bar{x} \beta_{1}(x)+Q_{a}\right)}\left(\bar{X} \beta_{1}(x)+Q_{a}\right)$

$$
\theta\left[R_{37}^{2} S_{x}^{2}+S_{y}^{2}\left(1-\rho^{2}\right)\right] \quad R_{37}=\frac{\beta_{1}(x) \bar{Y}}{\left(\beta_{1}(x) \bar{X}+Q_{a}\right)}
$$

Subramani et al (2014)

38. $\quad t_{38}=\frac{\hat{\bar{Y}}}{\left(\rho \bar{x}+Q_{1}\right)}\left(\rho \bar{X}+Q_{1}\right)$

$$
\theta\left[R_{38}^{2} S_{x}^{2}+S_{y}^{2}\left(1-\rho^{2}\right)\right] \quad R_{38}=\frac{\rho \bar{Y}}{\left(\rho \bar{X}+Q_{1}\right)}
$$

Subramani et al (2014)

39. $\quad t_{39}=\frac{\hat{\bar{Y}}}{\left(\rho \bar{x}+Q_{3}\right)}\left(\rho \bar{X}+Q_{3}\right)$

$$
\theta\left[R_{39}^{2} S_{x}^{2}+S_{y}^{2}\left(1-\rho^{2}\right)\right] \quad R_{39}=\frac{\rho \bar{Y}}{\left(\rho \bar{X}+Q_{3}\right)}
$$

Subramani et al (2014)

40. $\quad t_{40}=\frac{\hat{\bar{Y}}}{\left(\rho \bar{x}+Q_{r}\right)}\left(\rho \bar{X}+Q_{r}\right)$

Subramani et al (2014)

$$
\theta\left[R_{40}^{2} S_{x}^{2}+S_{y}^{2}\left(1-\rho^{2}\right)\right] \quad R_{40}=\frac{\rho \bar{Y}}{\left(\rho \bar{X}+Q_{r}\right)}
$$

41. $\quad t_{41}=\frac{\hat{\bar{Y}}}{\left(\rho \bar{x}+Q_{d}\right)}\left(\rho \bar{X}+Q_{d}\right)$

$$
\theta\left[R_{41}^{2} S_{x}^{2}+S_{y}^{2}\left(1-\rho^{2}\right)\right] \quad R_{41}=\frac{\rho \bar{Y}}{\left(\rho \bar{X}+Q_{d}\right)}
$$

Subramani et al (2014)

42. $\quad t_{42}=\frac{\hat{\bar{Y}}}{\left(\rho \bar{x}+Q_{a}\right)}\left(\rho \bar{X}+Q_{a}\right)$

$$
\theta\left[R_{42}^{2} S_{x}^{2}+S_{y}^{2}\left(1-\rho^{2}\right)\right] \quad R_{42}=\frac{\rho \bar{Y}}{\left(\rho \bar{X}+Q_{a}\right)}
$$

Subramani et al (2014)
43. $\quad t_{43}=\frac{\hat{\bar{Y}}}{\left(\bar{x}+T_{m}\right)}\left(\bar{X}+T_{m}\right)$

$$
\theta\left[R_{43}^{2} S_{x}^{2}+S_{y}^{2}\left(1-\rho^{2}\right)\right] \quad R_{43}=\frac{\bar{Y}}{\left(\bar{X}+T_{m}\right)}
$$

Abid et al (2016a)

$\begin{array}{ll}\text { 44. } & t_{44}=\frac{\hat{\bar{Y}}}{\left(\bar{x} C_{x}+T_{m}\right)}\left(\bar{X} C_{x}+T_{m}\right)\end{array} \quad \theta\left[R_{44}^{2} S_{x}^{2}+S_{y}^{2}\left(1-\rho^{2}\right)\right] \quad R_{44}=\frac{\bar{Y} C_{x}}{\left(\bar{X} C_{x}+T_{m}\right)}$


HOUSILA P. SINGH \& ANITA YADAV

Table 1. continued

S.No. Estimator
MSE of (.)

Population Ratio

45. $\quad t_{45}=\frac{\hat{\bar{Y}}}{\left(\bar{x} \rho+T_{m}\right)}\left(\bar{X} \rho+T_{m}\right)$

$$
\theta\left[R_{45}^{2} S_{x}^{2}+S_{y}^{2}\left(1-\rho^{2}\right)\right] \quad R_{45}=\frac{\bar{Y} \rho}{\left(\bar{X} \rho+T_{m}\right)}
$$

Abid et al (2016a)

46. $\quad t_{46}=\frac{\hat{\bar{Y}}}{\left(\bar{x}+M_{r}\right)}\left(\bar{X}+M_{r}\right)$ Abid et al (2016a)

47. $\quad t_{47}=\frac{\hat{\bar{Y}}}{\left(\bar{x} C_{x}+M_{r}\right)}\left(\bar{X} C_{x}+M_{r}\right) \quad \theta\left[R_{47}^{2} S_{x}^{2}+S_{y}^{2}\left(1-\rho^{2}\right)\right] \quad R_{47}=\frac{\bar{Y} C_{x}}{\left(\bar{X}+M_{r}\right)}$
Abid et al (2016a)

48. $\quad t_{48}=\frac{\hat{\bar{Y}}}{\left(\bar{x} \rho+M_{r}\right)}\left(\bar{X} \rho+M_{r}\right)$

$$
\theta\left[R_{46}^{2} S_{x}^{2}+S_{y}^{2}\left(1-\rho^{2}\right)\right] \quad R_{46}=\frac{\bar{Y}}{\left(\bar{X}+M_{r}\right)}
$$

Abid et al (2016a)

49. $\quad t_{49}=\frac{\hat{\bar{Y}}}{\left(\bar{x}+H_{l}\right)}\left(\bar{X}+H_{l}\right)$ Abid et al (2016a)

50. $\quad t_{50}=\frac{\hat{\bar{Y}}}{\left(\bar{x} C_{x}+H_{l}\right)}\left(\bar{X} C_{x}+H_{l}\right)$ Abid et al (2016a)
51. $\quad t_{51}=\frac{\hat{\bar{Y}}}{\left(\bar{x} \rho+H_{l}\right)}\left(\bar{X} \rho+H_{l}\right)$

$\theta\left[R_{48}^{2} S_{x}^{2}+S_{y}^{2}\left(1-\rho^{2}\right)\right] \quad R_{48}=\frac{\bar{Y} \rho}{\left(\bar{X} \rho+M_{r}\right)}$

Abid et al (2016a)

52. $t_{52}=\frac{\hat{\bar{Y}}}{(\bar{x}+G)}(\bar{X}+G)$ Abid et al (2016b)

53. $\quad t_{53}=\frac{\hat{\bar{Y}}}{(\bar{x} \rho+G)}(\bar{X} \rho+G)$ Abid et al (2016b)

54. $\quad t_{54}=\frac{\hat{\bar{Y}}}{\left(\bar{x} C_{x}+G\right)}\left(\bar{X} C_{x}+G\right) \quad \theta\left[R_{54}^{2} S_{x}^{2}+S_{y}^{2}\left(1-\rho^{2}\right)\right] \quad R_{54}=\frac{\bar{Y} C_{x}}{\left(\bar{X} C_{x}+G\right)}$ Abid et al (2016b)

$\begin{aligned} & \text { 55. } \quad t_{55}=\frac{\hat{\bar{Y}}}{(\bar{x}+D)}(\bar{X}+D) \\ & \text { Abid et al (2016b) }\end{aligned} \quad \theta\left[R_{55}^{2} S_{x}^{2}+S_{y}^{2}\left(1-\rho^{2}\right)\right] \quad R_{55}=\frac{\bar{Y}}{(\bar{X}+D)}$

Abid et al (2016b) 


\section{REDUCING THE MSE OF POPULATION ESTIMATORS}

Table 1. continued

S.No. Estimator
MSE of (.)

56. $\quad t_{56}=\frac{\hat{\bar{Y}}}{(\bar{x} \rho+D)}(\bar{X} \rho+D)$

$$
\theta\left[R_{56}^{2} S_{x}^{2}+S_{y}^{2}\left(1-\rho^{2}\right)\right] \quad R_{56}=\frac{\bar{Y} \rho}{(\bar{X} \rho+D)}
$$

Abid et al (2016b)

57. $\quad t_{57}=\frac{\hat{\bar{Y}}}{\left(\bar{x} C_{x}+D\right)}\left(\bar{X} C_{x}+D\right) \quad \theta\left[R_{57}^{2} S_{x}^{2}+S_{y}^{2}\left(1-\rho^{2}\right)\right] \quad R_{57}=\frac{\bar{Y} C_{x}}{\left(\bar{X} C_{x}+D\right)}$ Abid et al (2016b)

58. $\quad t_{58}=\frac{\hat{\bar{Y}}}{\left(\bar{x}+S_{p w}\right)}\left(\bar{X}+S_{p w}\right) \quad \theta\left[R_{58}^{2} S_{x}^{2}+S_{y}^{2}\left(1-\rho^{2}\right)\right] \quad R_{58}=\frac{\bar{Y} \rho}{\left(\bar{X}+S_{p w}\right)}$ Abid et al (2016b)

59. $\quad t_{59}=\frac{\hat{\bar{Y}}}{\left(\bar{x} \rho+S_{p w}\right)}\left(\bar{X} \rho+S_{p w}\right) \quad \theta\left[R_{59}^{2} S_{x}^{2}+S_{y}^{2}\left(1-\rho^{2}\right)\right] \quad R_{59}=\frac{\bar{Y} \rho}{\left(\bar{X} \rho+S_{p w}\right)}$

Abid et al (2016b)

60. $\quad t_{60}=\frac{\hat{\bar{Y}}}{\left(\bar{x} C_{x}+S_{p w}\right)}\left(\bar{X} C_{x}+S_{p w}\right) \quad \theta\left[R_{60}^{2} S_{x}^{2}+S_{y}^{2}\left(1-\rho^{2}\right)\right] \quad R_{60}=\frac{\bar{Y} C_{x}}{\left(\bar{X} C_{x}+S_{p w}\right)}$

Abid et al (2016b)

61. $\quad t_{61}=\frac{\hat{\bar{Y}}}{\left(\bar{x} \rho+D_{1}\right)}\left(\bar{X} \rho+D_{1}\right) \quad \theta\left[R_{61}^{2} S_{x}^{2}+S_{y}^{2}\left(1-\rho^{2}\right)\right] \quad R_{61}=\frac{\bar{Y} \rho}{\left(\bar{X} \rho+D_{1}\right)}$

Abid et al (2016c)

62. $\quad t_{62}=\frac{\hat{\bar{Y}}}{\left(\bar{x} \rho+D_{2}\right)}\left(\bar{X} \rho+D_{2}\right) \quad \theta\left[R_{62}^{2} S_{x}^{2}+S_{y}^{2}\left(1-\rho^{2}\right)\right] \quad R_{62}=\frac{\bar{Y} \rho}{\left(\bar{X} \rho+D_{2}\right)}$

Abid et al (2016c)

63. $\quad t_{63}=\frac{\hat{\bar{Y}}}{\left(\bar{x} \rho+D_{3}\right)}\left(\bar{X} \rho+D_{3}\right) \quad \theta\left[R_{63}^{2} S_{x}^{2}+S_{y}^{2}\left(1-\rho^{2}\right)\right] \quad R_{63}=\frac{\bar{Y} \rho}{\left(\bar{X} \rho+D_{3}\right)}$

Abid et al (2016c)

64. $\quad t_{64}=\frac{\hat{\bar{Y}}}{\left(\bar{x} \rho+D_{4}\right)}\left(\bar{X} \rho+D_{4}\right) \quad \theta\left[R_{64}^{2} S_{x}^{2}+S_{y}^{2}\left(1-\rho^{2}\right)\right] \quad R_{64}=\frac{\bar{Y} \rho}{\left(\bar{X} \rho+D_{4}\right)}$

Abid et al (2016c)

65. $\quad t_{65}=\frac{\hat{\bar{Y}}}{\left(\bar{x} \rho+D_{5}\right)}\left(\bar{X} \rho+D_{5}\right) \quad \theta\left[R_{65}^{2} S_{x}^{2}+S_{y}^{2}\left(1-\rho^{2}\right)\right] \quad R_{65}=\frac{\bar{Y} \rho}{\left(\bar{X} \rho+D_{5}\right)}$

Abid et al (2016c)
66. $\quad t_{66}=\frac{\hat{\bar{Y}}}{\left(\bar{x} \rho+D_{6}\right)}\left(\bar{X} \rho+D_{6}\right)$
$\theta\left[R_{66}^{2} S_{x}^{2}+S_{y}^{2}\left(1-\rho^{2}\right)\right] \quad R_{66}=\frac{\bar{Y} \rho}{\left(\bar{X} \rho+D_{6}\right)}$

Abid et al (2016c) 
HOUSILA P. SINGH \& ANITA YADAV

Table 1. continued

S.No. Estimator
MSE of (.)

Population Ratio

67. $\quad t_{67}=\frac{\hat{\bar{Y}}}{\left(\bar{x} \rho+D_{7}\right)}\left(\bar{X} \rho+D_{7}\right)$

$\theta\left[R_{67}^{2} S_{x}^{2}+S_{y}^{2}\left(1-\rho^{2}\right)\right] \quad R_{67}=\frac{\bar{Y} \rho}{\left(\bar{X} \rho+D_{7}\right)}$

Abid et al (2016c)

68. $\quad t_{68}=\frac{\hat{\bar{Y}}}{\left(\bar{x} \rho+D_{8}\right)}\left(\bar{X} \rho+D_{8}\right)$

$$
\theta\left[R_{68}^{2} S_{x}^{2}+S_{y}^{2}\left(1-\rho^{2}\right)\right] \quad R_{68}=\frac{\bar{Y} \rho}{\left(\bar{X} \rho+D_{8}\right)}
$$

Abid et al (2016c)

69. $\quad t_{69}=\frac{\hat{\bar{Y}}}{\left(\bar{x} \rho+D_{9}\right)}\left(\bar{X} \rho+D_{9}\right)$

$$
\theta\left[R_{69}^{2} S_{x}^{2}+S_{y}^{2}\left(1-\rho^{2}\right)\right] \quad R_{69}=\frac{\bar{Y} \rho}{\left(\bar{X} \rho+D_{9}\right)}
$$

Abid et al (2016c)

70. $\quad t_{70}=\frac{\hat{\bar{Y}}}{\left(\bar{x} \rho+D_{10}\right)}\left(\bar{X} \rho+D_{10}\right)$

$$
\theta\left[R_{70}^{2} S_{x}^{2}+S_{y}^{2}\left(1-\rho^{2}\right)\right] \quad R_{70}=\frac{\bar{Y} \rho}{\left(\bar{X} \rho+D_{10}\right)}
$$

Abid et al (2016c)

71. $\quad t_{71}=\frac{\hat{\bar{Y}}}{\left(\bar{x} C_{x}+D_{1}\right)}\left(\bar{X} C_{x}+D_{1}\right) \quad \theta\left[R_{71}^{2} S_{x}^{2}+S_{y}^{2}\left(1-\rho^{2}\right)\right] \quad R_{71}=\frac{\bar{Y} C_{x}}{\left(\bar{X} C_{x}+D_{1}\right)}$ Abid et al (2016c)

72. $\quad t_{72}=\frac{\hat{\bar{Y}}}{\left(\bar{x} C_{x}+D_{2}\right)}\left(\bar{X} C_{x}+D_{2}\right) \quad \theta\left[R_{72}^{2} S_{x}^{2}+S_{y}^{2}\left(1-\rho^{2}\right)\right] \quad R_{72}=\frac{\bar{Y} C_{x}}{\left(\bar{X} C_{x}+D_{2}\right)}$

Abid et al (2016c)

73. $\quad t_{73}=\frac{\hat{\bar{Y}}}{\left(\bar{x} C_{x}+D_{3}\right)}\left(\bar{X} C_{x}+D_{3}\right) \quad \theta\left[R_{73}^{2} S_{x}^{2}+S_{y}^{2}\left(1-\rho^{2}\right)\right] \quad R_{73}=\frac{\bar{Y} C_{x}}{\left(\bar{X} C_{x}+D_{3}\right)}$

Abid et al (2016c)

74. $\quad t_{74}=\frac{\hat{\bar{Y}}}{\left(\bar{x} C_{x}+D_{4}\right)}\left(\bar{X} C_{x}+D_{4}\right) \quad \theta\left[R_{74}^{2} S_{x}^{2}+S_{y}^{2}\left(1-\rho^{2}\right)\right] \quad R_{74}=\frac{\bar{Y} C_{x}}{\left(\bar{X} C_{x}+D_{4}\right)}$

Abid et al (2016c)

75. $\quad t_{75}=\frac{\hat{\bar{Y}}}{\left(\bar{x} C_{x}+D_{5}\right)}\left(\bar{X} C_{x}+D_{5}\right) \quad \theta\left[R_{75}^{2} S_{x}^{2}+S_{y}^{2}\left(1-\rho^{2}\right)\right] \quad R_{76}=\frac{\bar{Y} C_{x}}{\left(\bar{X} C_{x}+D_{5}\right)}$

Abid et al (2016c)

76. $\quad t_{76}=\frac{\hat{\bar{Y}}}{\left(\bar{x} C_{x}+D_{6}\right)}\left(\bar{X} C_{x}+D_{6}\right) \quad \theta\left[R_{76}^{2} S_{x}^{2}+S_{y}^{2}\left(1-\rho^{2}\right)\right] \quad R_{76}=\frac{\bar{Y} C_{x}}{\left(\bar{X} C_{x}+D_{6}\right)}$ Abid et al (2016c)

77. $\quad t_{77}=\frac{\hat{\bar{Y}}}{\left(\bar{x} C_{x}+D_{7}\right)}\left(\bar{X} C_{x}+D_{7}\right) \quad \theta\left[R_{77}^{2} S_{x}^{2}+S_{y}^{2}\left(1-\rho^{2}\right)\right] \quad R_{77}=\frac{\bar{Y} C_{x}}{\left(\bar{X} C_{x}+D_{7}\right)}$

Abid et al (2016c) 


\section{REDUCING THE MSE OF POPULATION ESTIMATORS}

Table 1. continued

\begin{tabular}{llcc}
\hline S.No. & Estimator & MSE of (.) & Population Ratio \\
\hline 78. & $t_{78}=\frac{\hat{\bar{Y}}}{\left(\bar{x} C_{x}+D_{8}\right)}\left(\bar{X} C_{x}+D_{8}\right)$ & $\theta\left[R_{78}^{2} S_{x}^{2}+S_{y}^{2}\left(1-\rho^{2}\right)\right]$ & $R_{78}=\frac{\bar{Y} C_{x}}{\left(\bar{X} C_{x}+D_{8}\right)}$ \\
& Abid et al (2016) & $\theta\left[R_{79}^{2} S_{x}^{2}+S_{y}^{2}\left(1-\rho^{2}\right)\right]$ & $R_{79}=\frac{\bar{Y} C_{x}}{\left(\bar{X} C_{x}+D_{9}\right)}$ \\
79. & $t_{79}=\frac{\hat{\bar{Y}}}{\left(\bar{x} C_{x}+D_{9}\right)}\left(\bar{X} C_{x}+D_{9}\right)$ & & \\
\hline 80. & Abid et al (2016c) & $\theta\left[R_{80}^{2} S_{x}^{2}+S_{y}^{2}\left(1-\rho^{2}\right)\right]$ & $R_{80}=\frac{\bar{Y} C_{x}}{\left(\bar{X} C_{x}+D_{10}\right)}$ \\
& Abid et al $(2016 \mathrm{c})$ & & \\
\hline
\end{tabular}

$$
E\left(e_{1} e_{3}\right)=\frac{N(N-n)}{(N-1)(N-2)} \frac{\mu_{30}}{n} \frac{1}{\bar{X} S_{x}^{2}}=\frac{(N-n)}{n(N-2)} \frac{\mu_{30}}{\bar{X} \mu_{20}},
$$

where $\mu_{r s}=E\left[\left(x_{i}-\bar{X}\right)^{r}\left(y_{i}-\bar{Y}\right)^{s}\right], C=\rho_{y x} \frac{C_{y}}{C_{x}}, C_{y}=\frac{S_{y}}{\bar{Y}}, C_{x}=\frac{S_{x}}{\bar{X}}$ and $\rho_{y x}=\frac{S_{x y}}{\left(S_{x} S_{y}\right)},(r, s)$ being non-negative integers.

Expressing ' $t$ ' defined by (6) in terms of e's

$$
t=\bar{Y}\left[1+e_{0}-\left(\frac{\beta \bar{X}}{\bar{Y}}\right) e_{1}\left(1+e_{2}\right)\left(1+e_{3}\right)^{-1}\right]\left(1+\tau e_{1}\right)^{-1} .
$$

where

$$
\tau=\frac{(a \bar{X})}{(a \bar{X}+b)} .
$$

Assume $\left|e_{1}\right|<1$ and $\left|e_{3}\right|<1$ so that we $\left(1+e_{3}\right)^{-1}$ and $\left(1+\tau e_{1}\right)^{-1}$ are expandable. Expanding the right hand side of (7), multiplying and neglecting terms of $e$ 's having power greater than two we have

$$
t=\bar{Y}\left[1+e_{0}-\tau e_{1}+\tau^{2} e_{1}^{2}-\tau e_{0} e_{1}-c\left(e_{1}+e_{1} e_{2}-e_{1} e_{3}-\tau e_{1}^{2}\right)\right]
$$




\section{HOUSILA P. SINGH \& ANITA YADAV}

Table 2. Some unknown members of the class of ratio type estimators $t$.

\begin{tabular}{|c|c|c|c|}
\hline \multirow{2}{*}{ S.No. } & \multirow{2}{*}{ Estimator } & \multicolumn{2}{|c|}{ Values of constants } \\
\hline & & $a$ & $\mathrm{~b}$ \\
\hline 1. & $t_{1}^{*}=\hat{\bar{Y}} \frac{\left(\beta_{1}(x) \bar{X}+\rho\right)}{\left(\beta_{1}(x) \bar{x}+\rho\right)}$ & $\beta_{1}(x)$ & $\rho$ \\
\hline 2. & $t_{2}^{*}=\hat{\bar{Y}} \frac{\left(\beta_{1}(x) \bar{X}+C_{x}\right)}{\left(\beta_{1}(x) \bar{x}+C_{x}\right)}$ & $\beta_{1}(x)$ & $C_{x}$ \\
\hline 3. & $t_{3}^{*}=\hat{\bar{Y}} \frac{\left(\bar{X} C_{x}+\beta_{1}(x)\right)}{\bar{x} C_{x}+\beta_{1}(x)}$ & $C_{x}$ & $\beta_{1}(x)$ \\
\hline 4. & $t_{4}^{*}=\hat{\bar{Y}}\left(\frac{\bar{X} \beta_{2}(x)+\beta_{1}(x)}{\bar{x} \beta_{2}(x)+\beta_{1}(x)}\right)$ & $\beta_{2}(x)$ & $\beta_{1}(x)$ \\
\hline 5. & $t_{5}^{*}=\hat{\bar{Y}}\left(\frac{\bar{X} \rho+\beta_{1}(x)}{\bar{x} \rho+\beta_{1}(x)}\right)$ & $\rho$ & $\beta_{1}(x)$ \\
\hline 7. & $t_{7}^{*}=\hat{\bar{Y}}\left(\frac{M_{d} \bar{X}+C_{x}}{\bar{x} M_{d}+C_{x}}\right)$ & $M_{d}$ & $C_{x}$ \\
\hline 8. & $t_{8}^{*}=\hat{\bar{Y}}\left(\frac{M_{d} \bar{X}+\beta_{2}(x)}{\bar{x} M_{d}+\beta_{2}(x)}\right)$ & $M_{d}$ & $\beta_{2}(x)$ \\
\hline 9. & $t_{9}^{*}=\hat{\bar{Y}}\left(\frac{\bar{X} M_{d}+\beta_{1}(x)}{\bar{x} M_{d}+\beta_{1}(x)}\right)$ & $M_{d}$ & $\beta_{1}(x)$ \\
\hline 10. & $t_{10}^{*}=\hat{\bar{Y}}\left(\frac{M_{d} \bar{X}+\rho}{\bar{x} M_{d}+\rho}\right)$ & $M_{d}$ & $\rho$ \\
\hline 11. & $t_{11}^{*}=\hat{\bar{Y}}\left(\frac{\bar{X}+Q_{d}}{\bar{x}+Q_{d}}\right)$ & 1 & $Q_{d}$ \\
\hline 12. & $t_{12}^{*}=\hat{\bar{Y}}\left(\frac{\bar{X} C_{x}+Q_{d}}{\bar{x} C_{x}+Q_{d}}\right)$ & $C_{x}$ & $Q_{d}$ \\
\hline 14. & $t_{14}^{*}=\hat{\bar{Y}}\left(\frac{\bar{X} M_{d}+Q_{d}}{\bar{x} M_{d}+Q_{d}}\right)$ & $M_{d}$ & $Q_{d}$ \\
\hline 15. & $t_{15}^{*}=\hat{\bar{Y}}\left(\frac{Q_{d} \bar{X}+C_{x}}{Q_{d} \bar{x}+C_{x}}\right)$ & $Q_{d}$ & $C_{x}$ \\
\hline 16. & $t_{16}^{*}=\hat{\bar{Y}}\left(\frac{Q_{d} \bar{X}+\beta_{2}(x)}{Q_{d} \bar{x}+\beta_{2}(x)}\right)$ & $Q_{d}$ & $\beta_{2}(x)$ \\
\hline
\end{tabular}




\section{REDUCING THE MSE OF POPULATION ESTIMATORS}

Table 2. continued

\begin{tabular}{|c|c|c|c|}
\hline \multirow{2}{*}{ S.No. } & \multirow{2}{*}{ Estimator } & \multicolumn{2}{|c|}{ Values of constants } \\
\hline & & $a$ & $\mathrm{~b}$ \\
\hline 17. & $t_{17}^{*}=\hat{\bar{Y}}\left(\frac{Q_{d} \bar{X}+\beta_{1}(x)}{Q_{d} \bar{x}+\beta_{1}(x)}\right)$ & $Q_{d}$ & $\beta_{1}(x)$ \\
\hline 18. & $t_{18}^{*}=\hat{\bar{Y}}\left(\frac{Q_{d} \bar{X}+\rho}{Q_{d} \bar{x}+\rho}\right)$ & $Q_{d}$ & $\rho$ \\
\hline 19. & $t_{19}^{*}=\hat{\bar{Y}}\left(\frac{Q_{d} \bar{X}+M_{d}}{Q_{d} \bar{x}+M_{d}}\right)$ & $Q_{d}$ & $M_{d}$ \\
\hline 20. & $\begin{array}{l}t_{20}^{*}=\hat{\bar{Y}}\left(\frac{\beta_{1}(x) \bar{X}+Q_{d}}{\beta_{1}(x) \bar{x}+Q_{d}}\right) \\
\text { Kumarapandiyan and } \\
\text { Subramani (2016)-type }\end{array}$ & $\beta_{1}(x)$ & $Q_{d}$ \\
\hline 21. & $t_{21}^{*}=\hat{\bar{Y}}\left(\frac{\bar{X} \beta_{2}(x)+T_{m}}{\bar{x} \beta_{2}(x)+T_{m}}\right)$ & $\beta_{2}(x)$ & $T_{m}$ \\
\hline 22. & $t_{22}^{*}=\hat{\bar{Y}}\left(\frac{\bar{X} \beta_{1}(x)+T_{m}}{\bar{x} \beta_{1}(x)+T_{m}}\right)$ & $\beta_{1}(x)$ & $T_{m}$ \\
\hline 23. & $t_{23}^{*}=\hat{\bar{Y}}\left(\frac{\bar{X} M_{d}+T_{m}}{\bar{x} M_{d}+T_{m}}\right)$ & $M_{d}$ & $T_{m}$ \\
\hline 24. & $t_{24}^{*}=\hat{\bar{Y}}\left(\frac{\bar{X} Q_{d}+T_{m}}{\bar{x} Q_{d}+T_{m}}\right)$ & $Q_{d}$ & $T_{m}$ \\
\hline 25. & $t_{25}^{*}=\hat{\bar{Y}}\left(\frac{\bar{X} T_{m}+C_{x}}{\bar{x} T_{m}+C_{x}}\right)$ & $T_{m}$ & $C_{x}$ \\
\hline 26. & $t_{26}^{*}=\hat{\bar{Y}}\left(\frac{\bar{X} T_{m}+\beta_{2}(x)}{\bar{x} T_{m}+\beta_{2}(x)}\right)$ & $T_{m}$ & $\beta_{2}(x)$ \\
\hline 27. & $t_{27}^{*}=\hat{\bar{Y}}\left(\frac{\bar{X} T_{m}+\rho}{\bar{x} T_{m}+\rho}\right)$ & $T_{m}$ & $\rho$ \\
\hline 28. & $t_{28}^{*}=\hat{\bar{Y}}\left(\frac{\bar{X} T_{m}+\beta_{1}(x)}{\bar{x} T_{m}+\beta_{1}(x)}\right)$ & $T_{m}$ & $\beta_{1}(x)$ \\
\hline 29. & $t_{29}^{*}=\hat{\bar{Y}}\left(\frac{\bar{X} T_{m}+M_{d}}{\bar{x} T_{m}+M_{d}}\right)$ & $T_{m}$ & $M_{d}$ \\
\hline
\end{tabular}


HOUSILA P. SINGH \& ANITA YADAV

Table 2. continued

\begin{tabular}{|c|c|c|c|}
\hline \multirow{2}{*}{ S.No. } & \multirow{2}{*}{ Estimator } & \multicolumn{2}{|c|}{ Values of constants } \\
\hline & & $a$ & $b$ \\
\hline 30. & $t_{30}^{*}=\hat{\bar{Y}}\left(\frac{\bar{X} T_{m}+Q_{d}}{\bar{x} T_{m}+Q_{d}}\right)$ & $T_{m}$ & $Q_{d}$ \\
\hline 31. & $t_{31}^{*}=\hat{\bar{Y}}\left(\frac{\bar{X} \beta_{2}(x)+M_{r}}{\bar{x} \beta_{2}(x)+M_{r}}\right)$ & $\beta_{2}(x)$ & $M_{r}$ \\
\hline 32. & $t_{32}^{*}=\hat{\bar{Y}}\left(\frac{\bar{X} \beta_{1}(x)+M_{r}}{\bar{x} \beta_{1}(x)+M_{r}}\right)$ & $\beta_{1}(x)$ & $M_{r}$ \\
\hline 33. & $t_{33}^{*}=\hat{\bar{Y}}\left(\frac{\bar{X} M_{d}+M_{r}}{\bar{x} M_{d}+M_{r}}\right)$ & $M_{d}$ & $M_{r}$ \\
\hline 34. & $t_{34}^{*}=\hat{\bar{Y}}\left(\frac{\bar{X} Q_{d}+M_{r}}{\bar{x} Q_{d}+M_{r}}\right)$ & $Q_{d}$ & $M_{r}$ \\
\hline 35. & $t_{35}^{*}=\hat{\bar{Y}}\left(\frac{\bar{X} M_{r}+C_{x}}{\bar{x} M_{r}+C_{x}}\right)$ & $M_{r}$ & $C_{x}$ \\
\hline 36. & $t_{36}^{*}=\hat{\bar{Y}}\left(\frac{\bar{X} M_{r}+\beta_{2}(x)}{\bar{x} M_{r}+\beta_{2}(x)}\right)$ & $M_{r}$ & $\beta_{2}(x)$ \\
\hline 37. & $t_{37}^{*}=\hat{\bar{Y}}\left(\frac{\bar{X} M_{r}+\rho}{\bar{x} M_{r}+\rho}\right)$ & $M_{r}$ & $\rho$ \\
\hline 38. & $t_{38}^{*}=\hat{\bar{Y}}\left(\frac{\bar{X} M_{r}+\beta_{1}(x)}{\bar{X} M_{r}+\beta_{1}(x)}\right)$ & $M_{r}$ & $\beta_{1}(x)$ \\
\hline 39. & $t_{39}^{*}=\hat{\bar{Y}}\left(\frac{\bar{X} M_{r}+M_{d}}{\bar{x} M_{r}+M_{d}}\right)$ & $M_{r}$ & $M_{d}$ \\
\hline 40. & $t_{40}^{*}=\hat{\bar{Y}}\left(\frac{\bar{X} M_{r}+Q_{d}}{\bar{x} M_{r}+Q_{d}}\right)$ & $M_{r}$ & $Q_{d}$ \\
\hline 41. & $t_{41}^{*}=\hat{\bar{Y}}\left(\frac{\bar{X} T_{m}+M_{r}}{\bar{x} T_{m}+M_{r}}\right)$ & $T_{m}$ & $M_{r}$ \\
\hline 42. & $t_{42}^{*}=\hat{\bar{Y}}\left(\frac{\bar{X} M_{r}+T_{m}}{\bar{x} M_{r}+T_{m}}\right)$ & $M_{r}$ & $T_{m}$ \\
\hline 43. & $t_{43}^{*}=\hat{\bar{Y}}\left(\frac{\bar{X} \beta_{2}(x)+H_{l}}{\bar{x} \beta_{2}(x)+H_{l}}\right)$ & $\beta_{2}(x)$ & $H_{l}$ \\
\hline
\end{tabular}


REDUCING THE MSE OF POPULATION ESTIMATORS

Table 2. continued

\begin{tabular}{|c|c|c|c|}
\hline \multirow{2}{*}{ S.No. } & \multirow{2}{*}{ Estimator } & \multicolumn{2}{|c|}{ Values of constants } \\
\hline & & a & $\mathrm{b}$ \\
\hline 44. & $t_{44}^{*}=\hat{\bar{Y}}\left(\frac{\bar{X} \beta_{1}(x)+H_{l}}{\bar{x} \beta_{1}(x)+H_{l}}\right)$ & $\beta_{1}(x)$ & $H_{l}$ \\
\hline 45. & $t_{45}^{*}=\hat{\bar{Y}}\left(\frac{\bar{X} M_{d}+H_{l}}{\bar{x} M_{d}+H_{l}}\right)$ & $M_{d}$ & $H_{l}$ \\
\hline 46. & $t_{46}^{*}=\hat{\bar{Y}}\left(\frac{\bar{X} Q_{d}+H_{l}}{\bar{x} Q_{d}+H_{l}}\right)$ & $Q_{d}$ & $H_{l}$ \\
\hline 47. & $t_{47}^{*}=\hat{\bar{Y}}\left(\frac{\bar{X} H_{l}+C_{x}}{\bar{x} H_{l}+C_{x}}\right)$ & $H_{l}$ & $C_{x}$ \\
\hline 48. & $t_{48}^{*}=\hat{\bar{Y}}\left(\frac{\bar{X} H_{l}+\beta_{2}(x)}{\bar{x} H_{l}+\beta_{2}(x)}\right)$ & $H_{l}$ & $\beta_{2}(x)$ \\
\hline 49. & $t_{49}^{*}=\hat{\bar{Y}}\left(\frac{\bar{X} H_{l}+\rho}{\bar{x} H_{l}+\rho}\right)$ & $H_{l}$ & $\rho$ \\
\hline 50. & $t_{50}^{*}=\hat{\bar{Y}}\left(\frac{\bar{X} H_{l}+\beta_{1}(x)}{\bar{x} H_{l}+\beta_{1}(x)}\right)$ & $H_{l}$ & $\beta_{1}(x)$ \\
\hline 51. & $t_{51}^{*}=\hat{\bar{Y}}\left(\frac{\bar{X} H_{l}+M_{d}}{\bar{x} H_{l}+M_{d}}\right)$ & $H_{l}$ & $M_{d}$ \\
\hline 52. & $t_{52}^{*}=\hat{\bar{Y}}\left(\frac{\bar{X} H_{l}+Q_{d}}{\bar{x} H_{l}+Q_{d}}\right)$ & $H_{l}$ & $Q_{d}$ \\
\hline 53. & $t_{53}^{*}=\hat{\bar{Y}}\left(\frac{\bar{X} H_{l}+M_{r}}{\bar{x} H_{l}+M_{r}}\right)$ & $H_{l}$ & $M_{r}$ \\
\hline 54. & $t_{54}^{*}=\hat{\bar{Y}}\left(\frac{\bar{X} T_{m}+H_{l}}{\bar{x} T_{m}+H_{l}}\right)$ & $T_{m}$ & $H_{l}$ \\
\hline 55. & $t_{55}^{*}=\hat{\bar{Y}}\left(\frac{\bar{X} H_{l}+T_{m}}{\bar{x} H_{l}+T_{m}}\right)$ & $H_{l}$ & $T_{m}$ \\
\hline 56. & $t_{56}^{*}=\hat{\bar{Y}}\left(\frac{\bar{X} M_{r}+H_{l}}{\bar{x} M_{r}+H_{l}}\right)$ & $M_{r}$ & $H_{l}$ \\
\hline 57. & $t_{57}^{*}=\hat{\bar{Y}}\left(\frac{\bar{X}+Q_{a}}{\bar{x}+Q_{a}}\right)$ & 1 & $Q_{a}$ \\
\hline
\end{tabular}


HOUSILA P. SINGH \& ANITA YADAV

Table 2. continued

\begin{tabular}{|c|c|c|c|}
\hline \multirow{2}{*}{ S.No. } & \multirow{2}{*}{ Estimator } & \multicolumn{2}{|c|}{ Values of constants } \\
\hline & & a & $\mathrm{b}$ \\
\hline 58. & $t_{58}^{*}=\hat{\bar{Y}}\left(\frac{\bar{X} C_{x}+Q_{a}}{\bar{x} C_{x}+Q_{a}}\right)$ & $C_{x}$ & $Q_{a}$ \\
\hline 62. & $t_{62}^{*}=\hat{\bar{Y}}\left(\frac{\bar{X} M_{d}+Q_{a}}{\bar{x} M_{d}+Q_{a}}\right)$ & $M_{d}$ & $Q_{a}$ \\
\hline 63. & $t_{63}^{*}=\hat{\bar{Y}}\left(\frac{\bar{X} Q_{d}+Q_{a}}{\bar{x} Q_{d}+Q_{a}}\right)$ & $Q_{d}$ & $Q_{a}$ \\
\hline 64. & $t_{64}^{*}=\hat{\bar{Y}}\left(\frac{\bar{X} Q_{d}+C_{x}}{\bar{x} Q_{d}+C_{x}}\right)$ & $Q_{d}$ & $C_{x}$ \\
\hline 65. & $t_{65}^{*}=\hat{\bar{Y}}\left(\frac{\bar{X} Q_{a}+\beta_{2}(x)}{\bar{x} Q_{a}+\beta_{2}(x)}\right)$ & $Q_{a}$ & $\beta_{2}(x)$ \\
\hline 66. & $t_{66}^{*}=\hat{\bar{Y}}\left(\frac{Q_{a} \bar{X}+\rho}{\bar{x} Q_{a}+\rho}\right)$ & $Q_{a}$ & $\rho$ \\
\hline 67. & $t_{67}^{*}=\hat{\bar{Y}}\left(\frac{Q_{a} \bar{X}+\beta_{1}(x)}{\bar{x} Q_{a}+\beta_{1}(x)}\right)$ & $Q_{a}$ & $\beta_{1}(x)$ \\
\hline 68. & $t_{68}^{*}=\hat{\bar{Y}}\left(\frac{Q_{a} \bar{X}+M_{d}}{\bar{x} Q_{a}+M_{d}}\right)$ & $Q_{a}$ & $M_{d}$ \\
\hline 69. & $t_{69}^{*}=\hat{\bar{Y}}\left(\frac{\bar{X} Q_{a}+Q_{d}}{\bar{x} Q_{a}+Q_{d}}\right)$ & $Q_{a}$ & $Q_{d}$ \\
\hline 70 & $t_{70}^{*}=\hat{\bar{Y}}\left(\frac{\bar{X} T_{m}+Q_{a}}{\bar{x} T_{m}+Q_{a}}\right)$ & $T_{m}$ & $Q_{a}$ \\
\hline 71. & $t_{71}^{*}=\hat{\bar{Y}}\left(\frac{\bar{X} Q_{a}+T_{m}}{\bar{x} Q_{a}+T_{m}}\right)$ & $Q_{a}$ & $T_{m}$ \\
\hline 72. & $t_{72}^{*}=\hat{\bar{Y}}\left(\frac{\bar{X} Q_{a}+M_{r}}{\bar{x} Q_{a}+M_{r}}\right)$ & $Q_{a}$ & $M_{r}$ \\
\hline 73. & $t_{73}^{*}=\hat{\bar{Y}}\left(\frac{\bar{X} M_{r}+Q_{a}}{\bar{x} M_{r}+Q_{a}}\right)$ & $M_{r}$ & $Q_{a}$ \\
\hline 74. & $t_{74}^{*}=\hat{\bar{Y}}\left(\frac{\bar{X} H_{l}+Q_{a}}{\bar{x} H_{l}+Q_{a}}\right)$ & $H_{l}$ & $Q_{a}$ \\
\hline
\end{tabular}


REDUCING THE MSE OF POPULATION ESTIMATORS

Table 2. continued

\begin{tabular}{|c|c|c|c|}
\hline \multirow{2}{*}{ S.No. } & \multirow{2}{*}{ Estimator } & \multicolumn{2}{|c|}{ Values of constants } \\
\hline & & $a$ & $\mathrm{~b}$ \\
\hline 75. & $t_{75}^{*}=\hat{\bar{Y}}\left(\frac{\bar{X} Q_{a}+H_{l}}{\bar{x} Q_{a}+H_{l}}\right)$ & $Q_{a}$ & $H_{l}$ \\
\hline 76. & $t_{76}^{*}=\hat{\bar{Y}}\left(\frac{\bar{X} Q_{r}+Q_{a}}{\bar{x} Q_{r}+Q_{a}}\right)$ & $Q_{r}$ & $Q_{a}$ \\
\hline 77. & $t_{77}^{*}=\hat{\bar{Y}}\left(\frac{\bar{X} Q_{a}+Q_{r}}{\bar{x} Q_{a}+Q_{r}}\right)$ & $Q_{a}$ & $Q_{r}$ \\
\hline 78. & $t_{78}^{*}=\hat{\bar{Y}}\left(\frac{\bar{X}+S_{x}}{\bar{x}+S_{x}}\right)$ & 1 & $S_{x}$ \\
\hline & Singh (2003)-type & & \\
\hline 79. & $t_{79}^{*}=\hat{\bar{Y}}\left(\frac{\bar{X} C_{x}+S_{x}}{\bar{x} C_{x}+S_{x}}\right)$ & $C_{x}$ & $S_{x}$ \\
\hline 80. & $t_{80}^{*}=\hat{\bar{Y}}\left(\frac{\bar{X} \beta_{2}(x)+S_{x}}{\bar{x} \beta_{2}(x)+S_{x}}\right)$ & $\beta_{2}(x)$ & $S_{x}$ \\
\hline & Singh (2003)-type & & \\
\hline 81. & $t_{81}^{*}=\hat{\bar{Y}}\left(\frac{\bar{X} \beta_{1}(x)+S_{x}}{\bar{x} \beta_{1}(x)+S_{x}}\right)$ & $\beta_{1}(x)$ & $S_{x}$ \\
\hline & Singh (2003) -type & & \\
\hline 82. & $t_{82}^{*}=\hat{\bar{Y}}\left(\frac{\bar{X} \rho+S_{x}}{\bar{x} \rho+S_{x}}\right)$ & $\rho$ & $S_{x}$ \\
\hline 83. & $t_{83}^{*}=\hat{\bar{Y}}\left(\frac{\bar{X} M_{d}+S_{x}}{\bar{x} M_{d}+S_{x}}\right)$ & $M_{d}$ & $S_{x}$ \\
\hline 84. & $t_{84}^{*}=\hat{\bar{Y}}\left(\frac{\bar{X} Q_{d}+Q_{a}}{\bar{x} Q_{d}+Q_{a}}\right)$ & $Q_{d}$ & $Q_{a}$ \\
\hline 85. & $t_{85}^{*}=\hat{\bar{Y}}\left(\frac{\bar{X} S_{x}+C_{x}}{\bar{x} S_{x}+C_{x}}\right)$ & $S_{x}$ & $C_{x}$ \\
\hline 86. & $t_{86}^{*}=\hat{\bar{Y}}\left(\frac{\bar{X} S_{x}+\beta_{2}(x)}{\bar{x} S_{x}+\beta_{2}(x)}\right)$ & $S_{x}$ & $\beta_{2}(x)$ \\
\hline 87. & $t_{87}^{*}=\hat{\bar{Y}}\left(\frac{\bar{X} S_{x}+\rho}{\bar{x} S_{x}+\rho}\right)$ & $S_{x}$ & $\rho$ \\
\hline
\end{tabular}


HOUSILA P. SINGH \& ANITA YADAV

Table 2. continued

\begin{tabular}{|c|c|c|c|}
\hline \multirow{2}{*}{ S.No. } & \multirow{2}{*}{ Estimator } & \multicolumn{2}{|c|}{ Values of constants } \\
\hline & & $a$ & $\mathrm{~b}$ \\
\hline 88. & $t_{88}^{*}=\hat{\bar{Y}}\left(\frac{\bar{X} S_{x}+\beta_{1}(x)}{\bar{x} S_{x}+\beta_{1}(x)}\right.$ & $S_{x}$ & $\beta_{1}(x)$ \\
\hline 89. & $t_{89}^{*}=\hat{\bar{Y}}\left(\frac{\bar{X} S_{x}+M_{d}}{\bar{x} S_{x}+M_{d}}\right)$ & $S_{x}$ & $M_{d}$ \\
\hline 90. & $t_{90}^{*}=\hat{\bar{Y}}\left(\frac{\bar{X} S_{x}+Q_{d}}{\bar{x} S_{x}+Q_{d}}\right)$ & $S_{x}$ & $Q_{d}$ \\
\hline 91. & $t_{91}^{*}=\hat{\bar{Y}}\left(\frac{\bar{X} T_{m}+S_{x}}{\bar{x} T_{m}+S_{x}}\right)$ & $T_{m}$ & $S_{x}$ \\
\hline 92. & $t_{92}^{*}=\hat{\bar{Y}}\left(\frac{\bar{X} S_{x}+T_{m}}{\bar{x} S_{x}+T_{m}}\right)$ & $S_{x}$ & $T_{m}$ \\
\hline 93. & $t_{93}^{*}=\hat{\bar{Y}}\left(\frac{\bar{X} S_{x}+M_{r}}{\bar{X} S_{x}+M_{r}}\right)$ & $S_{x}$ & $M_{r}$ \\
\hline 94. & $t_{94}^{*}=\hat{\bar{Y}}\left(\frac{\bar{X} M_{r}+S_{x}}{\bar{x} M_{r}+S_{x}}\right)$ & $M_{r}$ & $S_{x}$ \\
\hline 95. & $t_{95}^{*}=\hat{\bar{Y}}\left(\frac{\bar{X} H_{l}+S_{x}}{\bar{x} H_{l}+S_{x}}\right)$ & $H_{l}$ & $S_{x}$ \\
\hline 96. & $t_{96}^{*}=\hat{\bar{Y}}\left(\frac{\bar{X} S_{x}+H_{l}}{\bar{x} S_{x}+H_{l}}\right)$ & $S_{x}$ & $H_{l}$ \\
\hline 97. & $t_{97}^{*}=\hat{\bar{Y}}\left(\frac{\bar{X} Q_{r}+S_{x}}{\bar{x} Q_{r}+S_{x}}\right)$ & $Q_{r}$ & $S_{x}$ \\
\hline 98. & $t_{98}^{*}=\hat{\bar{Y}}\left(\frac{\bar{X} S_{x}+Q_{r}}{\bar{x} S_{x}+Q_{r}}\right)$ & $S_{x}$ & $Q_{r}$ \\
\hline 99. & $t_{99}^{*}=\hat{\bar{Y}}\left(\frac{\bar{X} Q_{a}+S_{x}}{\bar{x} Q_{a}+S_{x}}\right)$ & $Q_{a}$ & $S_{x}$ \\
\hline 100. & $t_{100}^{*}=\hat{\bar{Y}}\left(\frac{\bar{X} S_{x}+Q_{a}}{\bar{X} S_{x}+Q_{a}}\right)$ & $S_{x}$ & $Q_{a}$ \\
\hline 101. & $t_{101}^{*}=\hat{\bar{Y}}\left(\frac{\bar{X}+X}{\bar{x}+X}\right)$ & 1 & $X(=N \bar{X})$ \\
\hline
\end{tabular}




\section{REDUCING THE MSE OF POPULATION ESTIMATORS}

Table 2. continued

\begin{tabular}{|c|c|c|c|}
\hline \multirow{2}{*}{ S.No. } & \multirow{2}{*}{ Estimator } & \multicolumn{2}{|c|}{ Values of constants } \\
\hline & & $a$ & $\mathrm{~b}$ \\
\hline 102. & $t_{102}^{*}=\hat{\bar{Y}}\left(\frac{\bar{X} C_{x}+X}{\bar{x} C_{x}+X}\right)$ & $C_{x}$ & $X(=N \bar{X})$ \\
\hline 103. & $t_{103}^{*}=\hat{\bar{Y}}\left(\frac{\bar{X} \beta_{2}(x)+X}{\bar{x} \beta_{2}(x)+X}\right)$ & $\beta_{2}(x)$ & $X(=N \bar{X})$ \\
\hline 104. & $t_{104}^{*}=\hat{\bar{Y}}\left(\frac{\bar{X} \beta_{1}(x)+X}{\bar{x} \beta_{1}(x)+X}\right)$ & $\beta_{1}(x)$ & $X(=N \bar{X})$ \\
\hline 105. & $t_{105}^{*}=\hat{\bar{Y}}\left(\frac{\bar{X} \rho+X}{\bar{x} \rho+X}\right)$ & $\rho$ & $X(=N \bar{X})$ \\
\hline 102. & $t_{102}^{*}=\hat{\bar{Y}}\left(\frac{\bar{X} C_{x}+X}{\bar{x} C_{x}+X}\right)$ & $C_{x}$ & $X(=N \bar{X})$ \\
\hline 103. & $t_{103}^{*}=\hat{\bar{Y}}\left(\frac{\bar{X} \beta_{2}(x)+X}{\bar{x} \beta_{2}(x)+X}\right)$ & $\beta_{2}(x)$ & $X(=N \bar{X})$ \\
\hline 104. & $t_{104}^{*}=\hat{\bar{Y}}\left(\frac{\bar{X} \beta_{1}(x)+X}{\bar{x} \beta_{1}(x)+X}\right)$ & $\beta_{1}(x)$ & $X(=N \bar{X})$ \\
\hline 105. & $t_{105}^{*}=\hat{\bar{Y}}\left(\frac{\bar{X} \rho+X}{\bar{x} \rho+X}\right)$ & $\rho$ & $X(=N \bar{X})$ \\
\hline 106. & $t_{106}^{*}=\hat{\bar{Y}}\left(\frac{\bar{X} M_{d}+X}{\bar{x} M_{d}+X}\right)$ & $M_{d}$ & $X(=N \bar{X})$ \\
\hline 107. & $t_{107}^{*}=\hat{\bar{Y}}\left(\frac{\bar{X} Q_{d}+X}{\bar{x} Q_{d}+X}\right)$ & $Q_{d}$ & $X(=N \bar{X})$ \\
\hline 108. & $t_{108}^{*}=\hat{\bar{Y}}\left(\frac{\bar{X} X+C_{x}}{\bar{x} X+C_{x}}\right)$ & $X(=N \bar{X})$ & $C_{x}$ \\
\hline 109. & $t_{109}^{*}=\hat{\bar{Y}}\left(\frac{\bar{X} X+\beta_{2}(x)}{\bar{x} X+\beta_{2}(x)}\right)$ & $X(=N \bar{X})$ & $\beta_{2}(x)$ \\
\hline 110. & $t_{110}^{*}=\hat{\bar{Y}}\left(\frac{\bar{X} X+\rho}{\bar{x} X+\rho}\right)$ & $X(=N \bar{X})$ & $\rho$ \\
\hline 111. & $t_{111}^{*}=\hat{\bar{Y}}\left(\frac{\bar{X} X+\beta_{1}(x)}{\bar{x} X+\beta_{1}(x)}\right)$ & $X(=N \bar{X})$ & $\beta_{1}(x)$ \\
\hline
\end{tabular}


HOUSILA P. SINGH \& ANITA YADAV

Table 2. continued

\begin{tabular}{|c|c|c|c|}
\hline \multirow{2}{*}{ S.No. } & \multirow{2}{*}{ Estimator } & \multicolumn{2}{|c|}{ Values of constants } \\
\hline & & $a$ & $\mathrm{~b}$ \\
\hline 112. & $t_{112}^{*}=\hat{\bar{Y}}\left(\frac{\bar{X} X+M_{d}}{\bar{x} X+M_{d}}\right)$ & $X(=N \bar{X})$ & $M_{d}$ \\
\hline 113. & $t_{113}^{*}=\hat{\bar{Y}}\left(\frac{\bar{X} X+Q_{d}}{\bar{x} X+Q_{d}}\right)$ & $X(=N \bar{X})$ & $Q_{d}$ \\
\hline 114. & $t_{114}^{*}=\hat{\bar{Y}}\left(\frac{\bar{X} T_{m}+X}{\bar{x} T_{m}+X}\right)$ & $T_{m}$ & $X(=N \bar{X})$ \\
\hline 115. & $t_{115}^{*}=\hat{\bar{Y}}\left(\frac{\bar{X} X+T_{m}}{\bar{x} X+T_{m}}\right)$ & $X(=N \bar{X})$ & $T_{m}$ \\
\hline 116. & $t_{116}^{*}=\hat{\bar{Y}}\left(\frac{\bar{X} X+M_{r}}{\bar{x} X+M_{r}}\right)$ & $X(=N \bar{X})$ & $M_{r}$ \\
\hline 117. & $t_{117}^{*}=\hat{\bar{Y}}\left(\frac{\bar{X} M_{r}+X}{\bar{x} M_{r}+X}\right)$ & $M_{r}$ & $X(=N \bar{X})$ \\
\hline 118. & $t_{118}^{*}=\hat{\bar{Y}}\left(\frac{\bar{X} H_{l}+X}{\bar{x} H_{l}+X}\right)$ & $H_{l}$ & $X(=N \bar{X})$ \\
\hline 119. & $t_{119}^{*}=\hat{\bar{Y}}\left(\frac{\bar{X} X+H_{l}}{\bar{x} X+H_{l}}\right)$ & $X(=N \bar{X})$ & $H_{l}$ \\
\hline 120. & $t_{120}^{*}=\hat{\bar{Y}}\left(\frac{\bar{X} Q_{r}+X}{\bar{x} Q_{r}+X}\right)$ & $Q_{r}$ & $X(=N \bar{X})$ \\
\hline 121. & $t_{121}^{*}=\hat{\bar{Y}}\left(\frac{\bar{X} X+Q_{r}}{\bar{x} X+Q_{r}}\right)$ & $X(=N \bar{X})$ & $Q_{r}$ \\
\hline 122. & $t_{122}^{*}=\hat{\bar{Y}}\left(\frac{\bar{X} X+S_{x}}{\bar{x} X+S_{x}}\right)$ & $X(=N \bar{X})$ & $S_{x}$ \\
\hline 123. & $t_{123}^{*}=\hat{\bar{Y}}\left(\frac{\bar{X} S_{x}+X}{\bar{x} S_{x}+X}\right)$ & $S_{x}$ & $X(=N \bar{X})$ \\
\hline 124. & $t_{124}^{*}=\hat{\bar{Y}}\left(\frac{\bar{X} Q_{a}+X}{\bar{x} Q_{a}+X}\right)$ & $Q_{a}$ & $X(=N \bar{X})$ \\
\hline 125. & $t_{125}^{*}=\hat{\bar{Y}}\left(\frac{\bar{X} X+Q_{a}}{\bar{x} X+Q_{a}}\right)$ & $X(=N \bar{X})$ & $Q_{a}$ \\
\hline
\end{tabular}




\section{REDUCING THE MSE OF POPULATION ESTIMATORS}

Table 2. continued

\begin{tabular}{|c|c|c|c|}
\hline \multirow{2}{*}{ S.No. } & \multirow{2}{*}{ Estimator } & \multicolumn{2}{|c|}{ Values of constants } \\
\hline & & a & $\mathrm{b}$ \\
\hline 126. & $t_{126}^{*}=\hat{\bar{Y}}\left(\frac{\bar{X}+\Delta}{\bar{x}+\Delta}\right)$ & 1 & $\Delta$ \\
\hline 127. & $t_{127}^{*}=\hat{\bar{Y}}\left(\frac{\bar{X} C_{x}+\Delta}{\bar{x} C_{x}+\Delta}\right)$ & $C_{x}$ & $\Delta$ \\
\hline 128. & $t_{128}^{*}=\hat{\bar{Y}}\left(\frac{\bar{X} \beta_{2}(x)+\Delta}{\bar{x} \beta_{2}(x)+\Delta}\right)$ & $\beta_{2}(x)$ & $\Delta$ \\
\hline 129. & $t_{129}^{*}=\hat{\bar{Y}} \backslash\left(\frac{\bar{X} \beta_{1}(x)+\Delta}{\bar{x} \beta_{1}(x)+\Delta}\right)$ & $\beta_{1}(x)$ & $\Delta$ \\
\hline 130. & $t_{130}^{*}=\hat{\bar{Y}}\left(\frac{\bar{X} \rho+\Delta}{\bar{x} \rho+\Delta}\right)$ & $\rho$ & $\Delta$ \\
\hline 131. & $t_{131}^{*}=\hat{\bar{Y}}\left(\frac{\bar{X} M_{d}+\Delta}{\bar{x} M_{d}+\Delta}\right)$ & $M_{d}$ & $\Delta$ \\
\hline 132. & $t_{132}^{*}=\hat{\bar{Y}}\left(\frac{\bar{X} Q_{d}+\Delta}{\bar{x} Q_{d}+\Delta}\right)$ & $Q_{d}$ & $\Delta$ \\
\hline 133. & $t_{133}^{*}=\hat{\bar{Y}}\left(\frac{\bar{X} \Delta+C_{x}}{\bar{x} \Delta+C_{x}}\right)$ & $\Delta$ & $C_{x}$ \\
\hline 134. & $t_{134}^{*}=\hat{\bar{Y}}\left(\frac{\bar{X} \Delta+\beta_{2}(x)}{\bar{x} \Delta+\beta_{2}(x)}\right)$ & $\Delta$ & $\beta_{2}(x)$ \\
\hline 135. & $t_{135}^{*}=\hat{\bar{Y}}\left(\frac{\bar{X} \Delta+\rho}{\bar{x} \Delta+\rho}\right)$ & $\Delta$ & $\rho$ \\
\hline 136. & $t_{136}^{*}=\hat{\bar{Y}}\left(\frac{\bar{X} Q_{a}+\Delta}{\bar{x} Q_{a}+\Delta}\right)$ & $Q_{a}$ & $\Delta$ \\
\hline 137. & $t_{137}^{*}=\hat{\bar{Y}}\left(\frac{\bar{X} \Delta+Q_{a}}{\bar{x} \Delta+Q_{a}}\right)$ & $\Delta$ & $Q_{a}$ \\
\hline 138. & $t_{138}^{*}=\hat{\bar{Y}}\left(\frac{\bar{X} \Delta+S_{x}}{\bar{x} \Delta+S_{x}}\right)$ & $\Delta$ & $S_{x}$ \\
\hline 139. & $t_{139}^{*}=\hat{\bar{Y}}\left(\frac{\bar{X} S_{x}+\Delta}{\bar{x} S_{x}+\Delta}\right)$ & $S_{x}$ & $\Delta$ \\
\hline
\end{tabular}


HOUSILA P. SINGH \& ANITA YADAV

Table 2. continued

\begin{tabular}{|c|c|c|c|}
\hline \multirow{2}{*}{ S.No. } & \multirow{2}{*}{ Estimator } & \multicolumn{2}{|c|}{ Values of constants } \\
\hline & & a & b \\
\hline 140. & $t_{140}^{*}=\hat{\bar{Y}}\left(\frac{\bar{X} \Delta+\beta_{1}(x)}{\bar{x} \Delta+\beta_{1}(x)}\right)$ & $\Delta$ & $\beta_{1}(x)$ \\
\hline 141. & $t_{141}^{*}=\hat{\bar{Y}}\left(\frac{\bar{X} \Delta+M_{d}}{\bar{x} \Delta+M_{d}}\right)$ & $\Delta$ & $M_{d}$ \\
\hline 142. & $t_{142}^{*}=\hat{\bar{Y}}\left(\frac{\bar{X} \Delta+Q_{d}}{\bar{x} \Delta+Q_{d}}\right)$ & $\Delta$ & $Q_{d}$ \\
\hline 143. & $t_{143}^{*}=\hat{\bar{Y}}\left(\frac{\bar{X} T_{m}+\Delta}{\bar{x} T_{m}+\Delta}\right)$ & $T_{m}$ & $\Delta$ \\
\hline 144. & $t_{144}^{*}=\hat{\bar{Y}}\left(\frac{\bar{X} \Delta+T_{m}}{\bar{x} \Delta+T_{m}}\right)$ & $\Delta$ & $T_{m}$ \\
\hline 145. & $t_{145}^{*}=\hat{\bar{Y}}\left(\frac{\bar{X} \Delta+M_{r}}{\bar{x} \Delta+M_{r}}\right)$ & $\Delta$ & $M_{r}$ \\
\hline 146. & $t_{146}^{*}=\hat{\bar{Y}}\left(\frac{\bar{X} M_{r}+\Delta}{\bar{x} M_{r}+\Delta}\right)$ & $M_{r}$ & $\Delta$ \\
\hline 147. & $t_{147}^{*}=\hat{\bar{Y}}\left(\frac{\bar{X} H_{l}+\Delta}{\bar{x} H_{l}+\Delta}\right)$ & $H_{l}$ & $\Delta$ \\
\hline 148. & $t_{148}^{*}=\hat{\bar{Y}}\left(\frac{\bar{X} \Delta+H_{l}}{\bar{x} \Delta+H_{l}}\right)$ & $\Delta$ & $H_{l}$ \\
\hline 149. & $t_{149}^{*}=\hat{\bar{Y}}\left(\frac{\bar{X} Q_{r}+\Delta}{\bar{x} Q_{r}+\Delta}\right)$ & $Q_{r}$ & $\Delta$ \\
\hline 150. & $t_{150}^{*}=\hat{\bar{Y}}\left(\frac{\bar{X} \Delta+Q_{r}}{\bar{x} \Delta+Q_{r}}\right)$ & $\Delta$ & $Q_{r}$ \\
\hline 151. & $t_{151}^{*}=\hat{\bar{Y}}\left(\frac{\bar{X} \Delta+X}{\bar{x} \Delta+X}\right)$ & $\Delta$ & $X(=N \bar{X})$ \\
\hline 152. & $t_{152}^{*}=\hat{\bar{Y}}\left(\frac{\bar{X} X+\Delta}{\bar{x} X+\Delta}\right)$ & $X(=N \bar{X})$ & $\Delta$ \\
\hline 153. & $\begin{array}{l}t_{153}^{*}=\hat{\bar{Y}}\left(\frac{\bar{X}+Q_{1}}{\bar{x}+Q_{1}}\right) \\
\text { Al-Omar et al (2009) }\end{array}$ & 1 & $Q_{1}$ \\
\hline
\end{tabular}


REDUCING THE MSE OF POPULATION ESTIMATORS

Table 2. continued

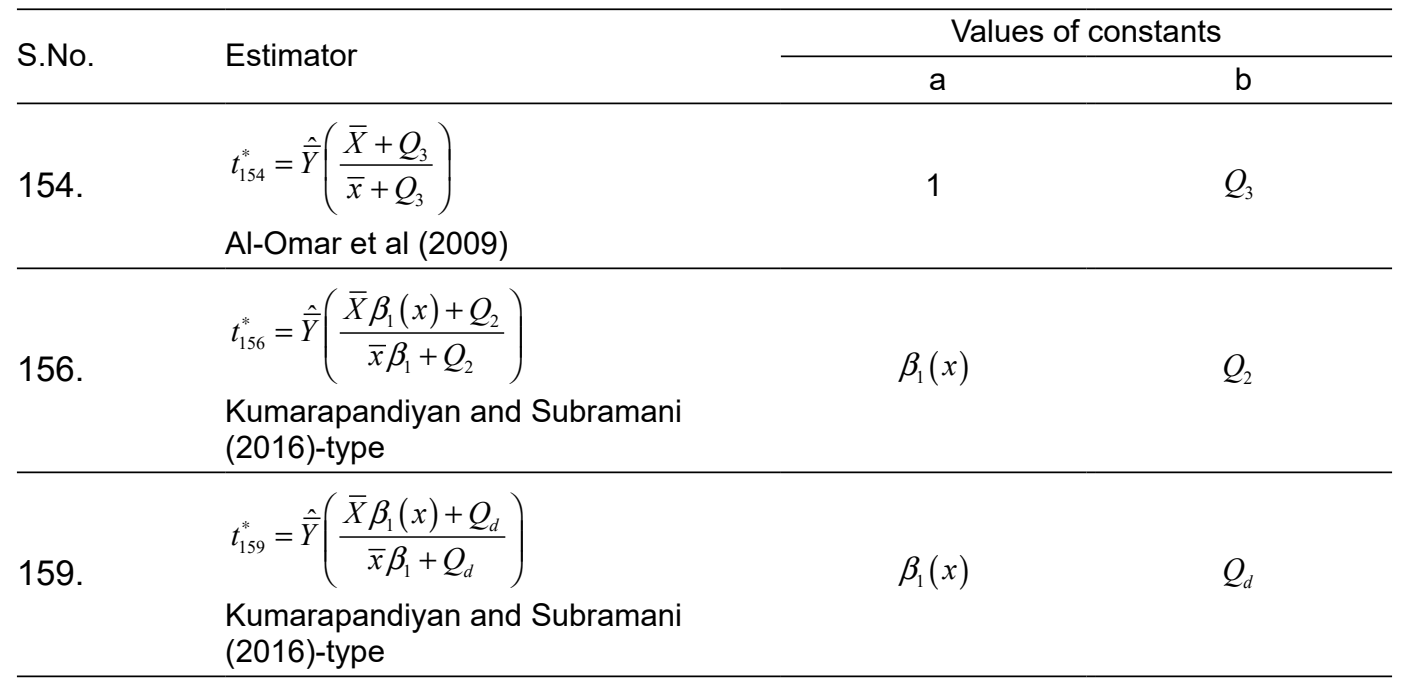

Or

$$
(t-\bar{Y})=\bar{Y}\left[e_{0}-\tau e_{1}+\tau^{2} e_{1}^{2}-\tau e_{0} e_{1}-c\left(e_{1}+e_{1} e_{2}-e_{1} e_{3}-\tau e_{1}^{2}\right)\right]
$$

Taking expectation of both sides of (2) we get the bias of ' $t$ ' to the the first degree of approximation as

$$
\begin{gathered}
B(t)=\frac{(1-f)}{n}\left[R_{J}^{2} \frac{S_{x}^{2}}{\bar{Y}}-\frac{N}{(N-2)} \beta\left(\frac{\mu_{21}}{\mu_{11}}-\frac{\mu_{30}}{\mu_{20}}\right)\right] \\
=\frac{(1-f)}{n}(A-B)
\end{gathered}
$$

where $\quad R_{J}=\frac{a \bar{Y}}{(a \bar{X}+b)}, A=R_{J}^{2}\left(\frac{S_{x}^{2}}{\bar{Y}}\right)$ and $B=\frac{N}{(N-2)} \beta\left(\frac{\mu_{21}}{\mu_{11}}-\frac{\mu_{30}}{\mu_{20}}\right)$.

The correct biases of the estimators listed in Table 1 and 2 can be obtained from (9) just by putting the suitable values of $(a, b)$. The biases of the estimators belonging to the class of estimators ' $\mathrm{t}$ ' is negligible if the sample size $\mathrm{n}$ is sufficiently large $($ i.e. $n \rightarrow N)$. It should be noted that the biases of the estimators $t_{1}$ to $t_{45}$ listed in Table 1 reported in Subramani and Kumarapandian (2012a,b,c,d) and Abid et al (2016a,b,c) are not correct. 
Squaring both sides of (8) and neglecting terms of e's having power greater than two

$$
(t-\bar{Y})=\bar{Y}^{2}\left[e_{0}^{2}-\tau^{2} e_{1}^{2}+C^{2} e_{1}^{2}-2 \tau e_{0} e_{1}-2 C e_{0} e+2 \tau C e_{1}^{2}\right]
$$

Taking expectation of both sides of (10), obtain the MSE of ' $t$ ' to the first degree of approximation as

$$
\operatorname{MSE}(t)=\frac{(1-f)}{n}\left[R_{J}^{2} S_{x}^{2}+S_{y}^{2}\left(1-\rho^{2}\right)\right]
$$

The MSE of the estimators belonging to class of estimators ' $t$ ' can be obtained from (11) just by putting the suitable values of $(a, b)$.

The proposed class of estimators ' $t$ ' is more efficient than the usual unbiased estimator $\bar{y}$ if

$$
\operatorname{MSE}(t)<\operatorname{MSE}(\bar{y})
$$

i.e. if

$$
R_{J}^{2} S_{x}^{2}<\beta^{2}
$$

The members of the proposed class of estimators ' $t$ ' is better than the usual unbiased estimator $\bar{y}$ as along as the condition (12) is satisfied. Further from (5) and (11)

$$
\operatorname{MSE}(t)<\operatorname{MSE}\left(\bar{y}_{r}\right)
$$

i.e. if

$$
R_{J}^{2}<(R-\beta)^{2}
$$

The members of the proposed class of estimators ' $t$ ' is more efficient than the usual ratio estimator $\bar{y}_{R}$ as long as the condition (13) is satisfied.

\section{Suggested Class of Ratio-Type Exponential Estimators}

Define a class of ratio-type exponential estimators for the population mean $\bar{Y}$ as

$$
t_{e}=\hat{\bar{Y}} \exp \left\{\frac{a(\bar{X}-\bar{x})}{a(\bar{X}+\bar{x})+2 b}\right\}
$$




\section{REDUCING THE MSE OF POPULATION ESTIMATORS}

$$
=\{\bar{y}+\hat{\beta}(\bar{X}-\bar{x})\} \exp \left\{\frac{a(\bar{X}-\bar{x})}{a(\bar{X}+\bar{x})+2 b}\right\},
$$

where $(a, b)$ are same as defined for the class of estimators ' $t$ ' at (1). A large number of estimators can be identified from the proposed class of estimators $t_{e}$ for suitable values of $(a, b)$. Some members of the proposed class of estimators $t_{e}$ corresponding to the members of the class of estimators $t$ are listed in Table 3.

Expressing $t_{e}$ in terms of e's we have

$$
t_{e}=\bar{Y}\left[1+e_{0}-C e_{1}\left(1+e_{2}\right)\left(1+e_{3}\right)^{-1}\right] \exp \left\{-\frac{\tau e_{1}}{2}\left(1+\frac{\tau}{2} e_{1}\right)^{-1}\right\}
$$

where,

$$
C=\left(\frac{\beta \bar{X}}{\bar{Y}}\right)=\rho \frac{C_{y}}{C_{x}} \text {. }
$$

Expanding the right hand side of (15), multiplying out and neglecting terms of e's having power greater than two we have

$$
t_{e}=\bar{Y}\left[1+e_{0}-\frac{\tau e_{1}}{2}-C\left(e_{1}+e_{1} e_{2}-e_{1} e_{3}\right)-\frac{\tau e_{0} e_{1}}{2}+\frac{\tau}{8}(3 \tau+4 C) e_{1}^{2}\right]
$$

or

$$
\left(t_{e}-\bar{Y}\right)=\bar{Y}\left[e_{0}-\frac{\tau e_{1}}{2}-C e_{1}-C\left(e_{1} e_{2}-e_{1} e_{3}\right)-\frac{\tau e_{0} e_{1}}{2}+\frac{\tau}{8}(3 \tau+4 C) e_{1}^{2}\right] .
$$

Taking expectation of both sides of (16) we get the bias of ' $t$ ' to the first degree of approximation, we have

$$
\begin{gathered}
B\left(t_{e}\right)=\frac{(1-f)}{n}\left[\frac{3}{8} R_{J}^{2}\left(\frac{S_{x}^{2}}{\bar{Y}}\right)-\frac{N}{(N-2)} \beta\left(\frac{\mu_{21}}{\mu_{11}}-\frac{\mu_{30}}{\mu_{20}}\right)\right], \\
=\frac{(1-f)}{n}\left(\frac{3}{8} A-B\right),
\end{gathered}
$$

where

$$
R_{J}=\frac{a \bar{Y}}{(a \bar{X}+b)}, \mathrm{A} \text { and } \mathrm{B} \text { are same as defined earlier. }
$$




\section{HOUSILA P. SINGH \& ANITA YADAV}

Table 3. Some members of the class of estimators $t_{e}$ corresponding to the estimators listed in Table 1.

\begin{tabular}{llll} 
& & \multicolumn{2}{c}{$\begin{array}{c}\text { Values of } \\
\text { Constants }\end{array}$} \\
S.No. & Estimators & MSE & $\mathrm{b}$ Population Ratio \\
\hline
\end{tabular}

1. $\quad t_{1 e}=\hat{\bar{Y}} \exp \left(\frac{\bar{X}-\bar{x}}{\bar{X}+\bar{x}}\right) \quad \theta\left[R_{1}^{2} \frac{S_{x}^{2}}{4}+S_{y}^{2}\left(1-\rho^{2}\right)\right] \quad 1 \quad 0 \quad R_{1}=\frac{\bar{Y}}{\bar{X}}=R$

2. $\quad t_{2 e}=\hat{\bar{Y}} \exp \left(\frac{\bar{X}-\bar{x}}{\bar{X}+\bar{x}+2 C_{x}}\right) \quad \theta\left[R_{2}^{2} \frac{S_{x}^{2}}{4}+S_{y}^{2}\left(1-\rho^{2}\right)\right] \quad 1 \quad C_{x} \quad R_{2}=\frac{\bar{Y}}{\left(\bar{X}+C_{x}\right)}$

3. $t_{3 e}=\hat{\bar{Y}} \exp \left(\frac{(\bar{X}-\bar{x})}{\bar{X}+\bar{x}+2 \beta_{2}(x)}\right) \quad \theta\left[R_{3}^{2} \frac{S_{x}^{2}}{4}+S_{y}^{2}\left(1-\rho^{2}\right)\right] \quad 1 \quad \beta_{2}(x) \quad R_{3}=\frac{\bar{Y}}{\bar{X}+\beta_{2}(x)}$

4. $\quad t_{4 e}=\hat{\bar{Y}} \exp \left(\frac{(\bar{X}-\bar{x}) \beta_{2}(x)}{\beta_{2}(x)(\bar{X}+\bar{x})+2 C_{x}}\right) \quad \theta\left[R_{4}^{2} \frac{S_{x}^{2}}{4}+S_{y}^{2}\left(1-\rho^{2}\right)\right] \quad \beta_{2}(x) \quad C_{x} \quad R_{4}=\frac{\bar{Y} \beta_{2}(x)}{\left(\bar{X} \beta_{2}(x)+C_{x}\right)}$

5. $\quad t_{5 e}=\hat{\bar{Y}} \exp \left(\frac{C_{x}(\bar{X}-\bar{x})}{C_{x}(\bar{X}+\bar{x})+2 \beta_{2}(x)}\right) \quad \theta\left[R_{5}^{2} \frac{S_{x}^{2}}{4}+S_{y}^{2}\left(1-\rho^{2}\right)\right] \quad C_{x} \quad \beta_{2}(x) \quad R_{5}=\frac{\bar{Y} C_{x}}{\left(\bar{X} C_{x}+\beta_{2}(x)\right)}$

6. $\quad t_{6 e}=\hat{\bar{Y}} \exp \left(\frac{(\bar{X}-\bar{x})}{\bar{X}+\bar{x}+2 \rho}\right) \quad \theta\left[R_{6}^{2} \frac{S_{x}^{2}}{4}+S_{y}^{2}\left(1-\rho^{2}\right)\right] \quad 1 \quad \rho \quad R_{6}=\frac{\bar{Y}}{\bar{X}+\rho}$

7. $\quad t_{7 e}=\hat{\bar{Y}} \exp \left(\frac{C_{x}(\bar{X}-\bar{x})}{C_{x}(\bar{X}+\bar{x})+2 \rho}\right) \quad \theta\left[R_{7}^{2} \frac{S_{x}^{2}}{4}+S_{y}^{2}\left(1-\rho^{2}\right)\right] \quad C_{x} \quad \rho \quad R_{7}=\frac{\bar{Y} C_{x}}{\left(\bar{X} C_{x}+\rho\right)}$

8. $\quad t_{8 e}=\hat{\bar{Y}} \exp \left(\frac{\rho(\bar{X}-\bar{x})}{\rho(\bar{X}+\bar{x})+2 C_{x}}\right) \quad \theta\left[R_{8}^{2} \frac{S_{x}^{2}}{4}+S_{y}^{2}\left(1-\rho^{2}\right)\right] \quad \rho \quad C_{x} \quad R_{8}=\frac{\bar{Y} \rho}{\left(\bar{X} \rho+C_{x}\right)}$

9. $\quad t_{9 e}=\hat{\bar{Y}} \exp \left(\frac{\beta_{2}(x)(\bar{X}-\bar{x})}{\beta_{2}(x)(\bar{X}+\bar{x})+2 \rho}\right) \quad \theta\left[R_{9}^{2} \frac{S_{x}^{2}}{4}+S_{y}^{2}\left(1-\rho^{2}\right)\right] \beta_{2}(x) \quad \rho \quad R_{9}=\frac{\bar{Y} \beta_{2}(x)}{\left(\bar{X} \beta_{2}(x)+\rho\right)}$

10. $t_{10 e}=\hat{\bar{Y}} \exp \left(\frac{\rho(\bar{X}-\bar{x})}{\rho(\bar{X}+\bar{x})+2 \beta_{2}(x)}\right) \quad \theta\left[R_{10}^{2} \frac{S_{x}^{2}}{4}+S_{y}^{2}\left(1-\rho^{2}\right)\right] \quad \rho \quad \beta_{2}(x) \quad R_{10}=\frac{\bar{Y} \rho}{\left(\bar{X} \rho+\beta_{2}(x)\right)}$

11. $t_{11 e}=\hat{\bar{Y}} \exp \left(\frac{(\bar{X}-\bar{x})}{(\bar{X}+\bar{x})+2 \beta_{1}(x)}\right) \quad \theta\left[R_{11}^{2} \frac{S_{x}^{2}}{4}+S_{y}^{2}\left(1-\rho^{2}\right)\right] \quad 1 \quad \beta_{2}(x) \quad R_{11}=\frac{\bar{Y}}{\left(\bar{X}+\beta_{1}(x)\right)}$ 


\section{REDUCING THE MSE OF POPULATION ESTIMATORS}

Table 3. continued

\begin{tabular}{|c|c|c|c|c|}
\hline \multirow[b]{2}{*}{ S.No. Estimators } & \multirow[b]{2}{*}{ MSE } & \multicolumn{2}{|c|}{$\begin{array}{l}\text { Values of } \\
\text { Constants }\end{array}$} & \multirow[b]{2}{*}{ Population Ratio } \\
\hline & & $a$ & $\mathrm{~b}$ & \\
\hline 12. $t_{12 e}=\hat{\bar{Y}} \exp \left(\frac{\beta_{1}(x)(\bar{X}-\bar{x})}{\beta_{1}(x)(\bar{X}+\bar{x})+2 \beta_{2}(x)}\right.$ & $\theta\left[R_{12}^{2} \frac{S_{x}^{2}}{4}+S_{y}^{2}\left(1-\rho^{2}\right)\right]$ & $\beta_{1}(x)$ & $\beta_{2}(x)$ & $R_{12}=\frac{\bar{Y} \beta_{1}(x)}{\left(\bar{X} \beta_{1}(x)+\beta_{2}(x)\right)}$ \\
\hline 13. $t_{13 e}=\hat{\bar{Y}} \exp \left(\frac{(\bar{X}-\bar{x})}{(\bar{X}+\bar{x})+2 M_{d}}\right.$ & $\theta\left[R_{13}^{2} \frac{S_{x}^{2}}{4}+S_{y}^{2}\left(1-\rho^{2}\right)\right.$ & 1 & $M_{d}$ & $R_{13}=\frac{\bar{Y}}{\left(\bar{X}+M_{d}\right)}$ \\
\hline 14. $t_{14 e}=\hat{\bar{Y}} \exp \left(\frac{C_{x}(\bar{X}-\bar{x})}{C_{x}(\bar{X}+\bar{x})+2 M_{d}}\right.$ & $\theta\left[R_{14}^{2} \frac{S_{x}^{2}}{4}+S_{y}^{2}\left(1-\rho^{2}\right)\right]$ & $C_{x}$ & $M_{d}$ & $R_{14}=\frac{\bar{Y} C_{x}}{\left(\bar{X} C_{x}+M_{d}\right)}$ \\
\hline 15. $t_{15 e}=\hat{\bar{Y}} \exp \left(\frac{\beta_{1}(x)(\bar{X}-\bar{x})}{\beta_{1}(x)(\bar{X}+\bar{x})+2 M_{d}}\right.$ & $\theta\left[R_{15}^{2} \frac{S_{x}^{2}}{4}+S_{y}^{2}\left(1-\rho^{2}\right)\right]$ & $\beta_{1}(x)$ & $M_{d}$ & $R_{15}=\frac{\beta_{1}(x) \bar{Y}}{\left(\bar{X} \beta_{1}(x)+M_{d}\right)}$ \\
\hline 16. $t_{16 e}=\hat{\bar{Y}} \exp \left(\frac{\beta_{2}(x)(\bar{X}-\bar{x})}{\beta_{2}(x)(\bar{X}+\bar{x})+2 M_{d}}\right)$ & $\theta\left[R_{16}^{2} \frac{S_{x}^{2}}{4}+S_{y}^{2}\left(1-\rho^{2}\right)\right]$ & $\beta_{2}(x)$ & $M_{d}$ & $R_{16}=\frac{\beta_{2}(x) \bar{Y}}{\left(\bar{X} \beta_{2}(x)+M_{d}\right)}$ \\
\hline 17. $t_{17 e}=\hat{\bar{Y}} \exp \left(\frac{(\bar{X}-\bar{x})}{(\bar{X}+\bar{x})+2 D_{1}}\right.$ & $\theta\left[R_{17}^{2} \frac{S_{x}^{2}}{4}+S_{y}^{2}\left(1-\rho^{2}\right)\right]$ & 1 & $D_{1}$ & $R_{17}=\frac{\bar{Y}}{\left(\bar{X}+D_{1}\right)}$ \\
\hline 18. $t_{18 e}=\hat{\bar{Y}} \exp \left(\frac{(\bar{X}-\bar{x})}{(\bar{X}+\bar{x})+2 D_{2}}\right.$ & $\theta\left[R_{18}^{2} \frac{S_{x}^{2}}{4}+S_{y}^{2}\left(1-\rho^{2}\right)\right]$ & 1 & $D_{2}$ & $R_{18}=\frac{\bar{Y}}{\left(\bar{X}+D_{2}\right)}$ \\
\hline$t_{19 e}=\hat{\bar{Y}} \exp \left(\frac{(\bar{X}-\bar{x})}{(\bar{X}+\bar{x})+2 D_{3}}\right.$ & $\theta\left[R_{19}^{2} \frac{S_{x}^{2}}{4}+S_{y}^{2}\left(1-\rho^{2}\right)\right]$ & 1 & $D_{3}$ & $R_{19}=\frac{\bar{Y}}{\left(\bar{X}+D_{3}\right)}$ \\
\hline$t_{20 e}=\hat{\bar{Y}} \exp \left(\frac{(\bar{X}-\bar{x})}{(\bar{X}+\bar{x})+2 D_{4}}\right)$ & $\theta\left[R_{20}^{2} \frac{S_{x}^{2}}{4}+S_{y}^{2}\left(1-\rho^{2}\right)\right.$ & 1 & $D_{4}$ & $R_{20}=\frac{\bar{Y}}{\left(\bar{X}+D_{4}\right)}$ \\
\hline$t_{21 e}=\hat{\bar{Y}} \exp \left(\frac{(\bar{X}-\bar{x})}{(\bar{X}+\bar{x})+2 D_{5}}\right.$ & $\theta\left[R_{21}^{2} \frac{S_{x}^{2}}{4}+S_{y}^{2}\left(1-\rho^{2}\right)\right]$ & 1 & $D_{5}$ & $R_{21}=\frac{\bar{Y}}{\left(\bar{X}+D_{5}\right)}$ \\
\hline$t_{22 e}=\hat{\bar{Y}} \exp \left(\frac{(\bar{X}-\bar{x})}{(\bar{X}+\bar{x})+2 D_{6}}\right)$ & $\theta\left[R_{22}^{2} \frac{S_{x}^{2}}{4}+S_{y}^{2}\left(1-\rho^{2}\right)\right.$ & 1 & $D_{6}$ & $R_{22}=\frac{\bar{Y}}{\left(\bar{X}+D_{6}\right)}$ \\
\hline 23. $t_{23 e}=\hat{\bar{Y}} \exp \left(\frac{(\bar{X}-\bar{x})}{(\bar{X}+\bar{x})+2 D_{7}}\right.$ & $\theta\left[R_{23}^{2} \frac{S_{x}^{2}}{4}+S_{y}^{2}\left(1-\rho^{2}\right)\right]$ & 1 & $D_{7}$ & $R_{23}=\frac{\bar{Y}}{\left(\bar{X}+D_{7}\right)}$ \\
\hline
\end{tabular}


HOUSILA P. SINGH \& ANITA YADAV

Table 3. continued

\begin{tabular}{|c|c|c|c|c|c|c|}
\hline \multirow{2}{*}{\multicolumn{3}{|c|}{ S.No. Estimators }} & \multirow[b]{2}{*}{ MSE } & \multicolumn{2}{|c|}{$\begin{array}{l}\text { Values of } \\
\text { Constants }\end{array}$} & \multirow[b]{2}{*}{ Population Ratio } \\
\hline & & & & $a$ & $\mathrm{~b}$ & \\
\hline 24. & $t_{24 e}=\hat{\bar{Y}} \exp$ & $\mathrm{p}\left(\frac{(\bar{X}-\bar{x})}{(\bar{X}+\bar{x})+2 D_{8}}\right.$ & $\theta\left[R_{24}^{2} \frac{S_{x}^{2}}{4}+S_{y}^{2}\left(1-\rho^{2}\right)\right]$ & 1 & $D_{8}$ & $R_{24}=\frac{\bar{Y}}{\left(\bar{X}+D_{8}\right)}$ \\
\hline 25. & $t_{25 e}=\hat{\bar{Y}} \exp$ & $\mathrm{p}\left(\frac{(\bar{X}-\bar{x})}{(\bar{X}+\bar{x})+2 D_{9}}\right)$ & $\theta\left[R_{25}^{2} \frac{S_{x}^{2}}{4}+S_{y}^{2}\left(1-\rho^{2}\right)\right]$ & 1 & $D_{9}$ & $R_{25}=\frac{\bar{Y}}{\left(\bar{X}+D_{9}\right)}$ \\
\hline 26. & $t_{26 e}=\hat{\bar{Y}} \exp$ & $\mathrm{p}\left(\frac{(\bar{X}-\bar{x})}{(\bar{X}+\bar{x})+2 D_{10}}\right.$ & $\theta\left[R_{26}^{2} \frac{S_{x}^{2}}{4}+S_{y}^{2}\left(1-\rho^{2}\right)\right]$ & 1 & $D_{10}$ & $R_{26}=\frac{\bar{Y}}{\left(\bar{X}+D_{10}\right)}$ \\
\hline 27. & $t_{27 e}=\hat{\bar{Y}} \exp \left(\frac{-}{\beta}\right.$ & $\frac{\beta_{1}(x)(\bar{X}-\bar{x})}{\beta_{1}(x)(\bar{X}+\bar{x})+2 Q_{d}}$ & $\theta\left[R_{27}^{2} \frac{S_{x}^{2}}{4}+S_{y}^{2}\left(1-\rho^{2}\right)\right]$ & $\beta_{1}(x)$ & $Q_{d}$ & $R_{27}=\frac{\bar{Y} \beta_{1}(x)}{\left(\bar{X} \beta_{1}(x)+Q_{d}\right)}$ \\
\hline 28. & $t_{28 e}=\hat{\bar{Y}} \exp ($ & $\left(\frac{\rho(\bar{X}-\bar{x})}{\rho(\bar{X}+\bar{x})+2 M_{d}}\right.$ & $\theta\left[R_{28}^{2} \frac{S_{x}^{2}}{4}+S_{y}^{2}\left(1-\rho^{2}\right)\right]$ & $\rho$ & $M_{d}$ & $R_{28}=\frac{\rho \bar{Y}}{\left(\rho \bar{X}+M_{d}\right)}$ \\
\hline 29. & $t_{29 e}=\hat{\bar{Y}} \exp \left(\frac{-}{\beta}\right.$ & $\frac{\beta_{2}(x)(\bar{X}-\bar{x})}{\beta_{2}(x)(\bar{X}+\bar{x})+2 Q_{1}}$ & $\theta\left[R_{29}^{2} \frac{S_{x}^{2}}{4}+S_{y}^{2}\left(1-\rho^{2}\right)\right]$ & $\beta_{2}(x)$ & $Q_{1}$ & $R_{29}=\frac{\bar{Y} \beta_{2}(x)}{\left(\bar{X} \beta_{2}(x)+Q_{1}\right)}$ \\
\hline
\end{tabular}

30. $t_{30 e}=\hat{\bar{Y}} \exp \left(\frac{\beta_{2}(x)(\bar{X}-\bar{x})}{\beta_{2}(x)(\bar{X}+\bar{x})+2 Q_{3}}\right) \quad \theta\left[R_{30}^{2} \frac{S_{x}^{2}}{4}+S_{y}^{2}\left(1-\rho^{2}\right)\right] \quad \beta_{2}(x) \quad Q_{3} \quad R_{30}=\frac{\bar{Y} \beta_{2}(x)}{\left(\bar{X} \beta_{2}(x)+Q_{3}\right)}$

31. $t_{31 e}=\hat{\bar{Y}} \exp \left(\frac{\beta_{2}(x)(\bar{X}-\bar{x})}{\beta_{2}(x)(\bar{X}+\bar{x})+2 Q_{r}}\right) \quad \theta\left[R_{31}^{2} \frac{S_{x}^{2}}{4}+S_{y}^{2}\left(1-\rho^{2}\right)\right] \beta_{2}(x) \quad Q_{r} \quad R_{31}=\frac{\bar{Y} \beta_{2}(x)}{\left(\bar{X} \beta_{2}(x)+Q_{r}\right)}$

32. $t_{32 e}=\hat{\bar{Y}} \exp \left(\frac{\beta_{2}(x)(\bar{X}-\bar{x})}{\beta_{2}(x)(\bar{X}+\bar{x})+2 Q_{d}}\right) \quad \theta\left[R_{32}^{2} \frac{S_{x}^{2}}{4}+S_{y}^{2}\left(1-\rho^{2}\right)\right] \beta_{2}(x) \quad Q_{d} \quad R_{32}=\frac{\bar{Y} \beta_{2}(x)}{\left(\bar{X} \beta_{2}(x)+Q_{d}\right)}$

33. $t_{33 e}=\hat{\bar{Y}} \exp \left(\frac{\beta_{2}(x)(\bar{X}-\bar{x})}{\beta_{2}(x)(\bar{X}+\bar{x})+2 Q_{a}}\right) \quad \theta\left[R_{33}^{2} \frac{S_{x}^{2}}{4}+S_{y}^{2}\left(1-\rho^{2}\right)\right] \quad \beta_{2}(x) \quad Q_{a} \quad R_{33}=\frac{\bar{Y} \beta_{2}(x)}{\left(\bar{X} \beta_{2}(x)+Q_{a}\right)}$

34. $t_{34 e}=\hat{\bar{Y}} \exp \left(\frac{\beta_{1}(x)(\bar{X}-\bar{x})}{\beta_{1}(x)(\bar{X}+\bar{x})+2 Q_{1}}\right) \quad \theta\left[R_{34}^{2} \frac{S_{x}^{2}}{4}+S_{y}^{2}\left(1-\rho^{2}\right)\right] \quad \beta_{1}(x) \quad Q_{1} \quad R_{34}=\frac{\bar{Y} \beta_{1}(x)}{\left(\bar{X} \beta_{1}(x)+Q_{1}\right)}$

35. $t_{35 e}=\hat{\bar{Y}} \exp \left(\frac{\beta_{1}(x)(\bar{X}-\bar{x})}{\beta_{1}(x)(\bar{X}+\bar{x})+2 Q_{3}}\right) \quad \theta\left[R_{35}^{2} \frac{S_{x}^{2}}{4}+S_{y}^{2}\left(1-\rho^{2}\right)\right] \quad \beta_{1}(x) \quad Q_{3} \quad R_{35}=\frac{\bar{Y} \beta_{1}(x)}{\left(\bar{X} \beta_{1}(x)+Q_{3}\right)}$ 


\section{REDUCING THE MSE OF POPULATION ESTIMATORS}

Table 3. continued

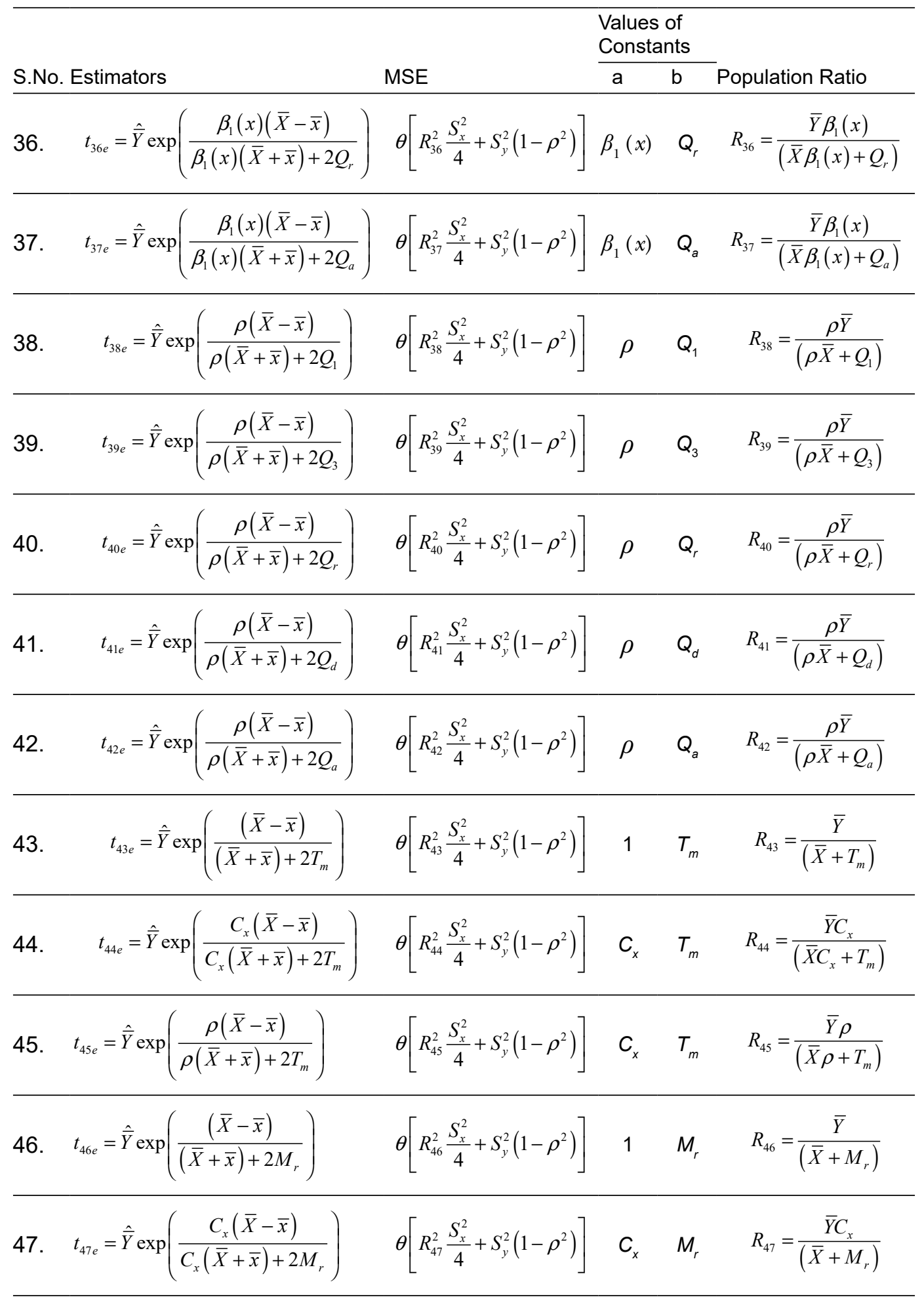


HOUSILA P. SINGH \& ANITA YADAV

Table 3. continued

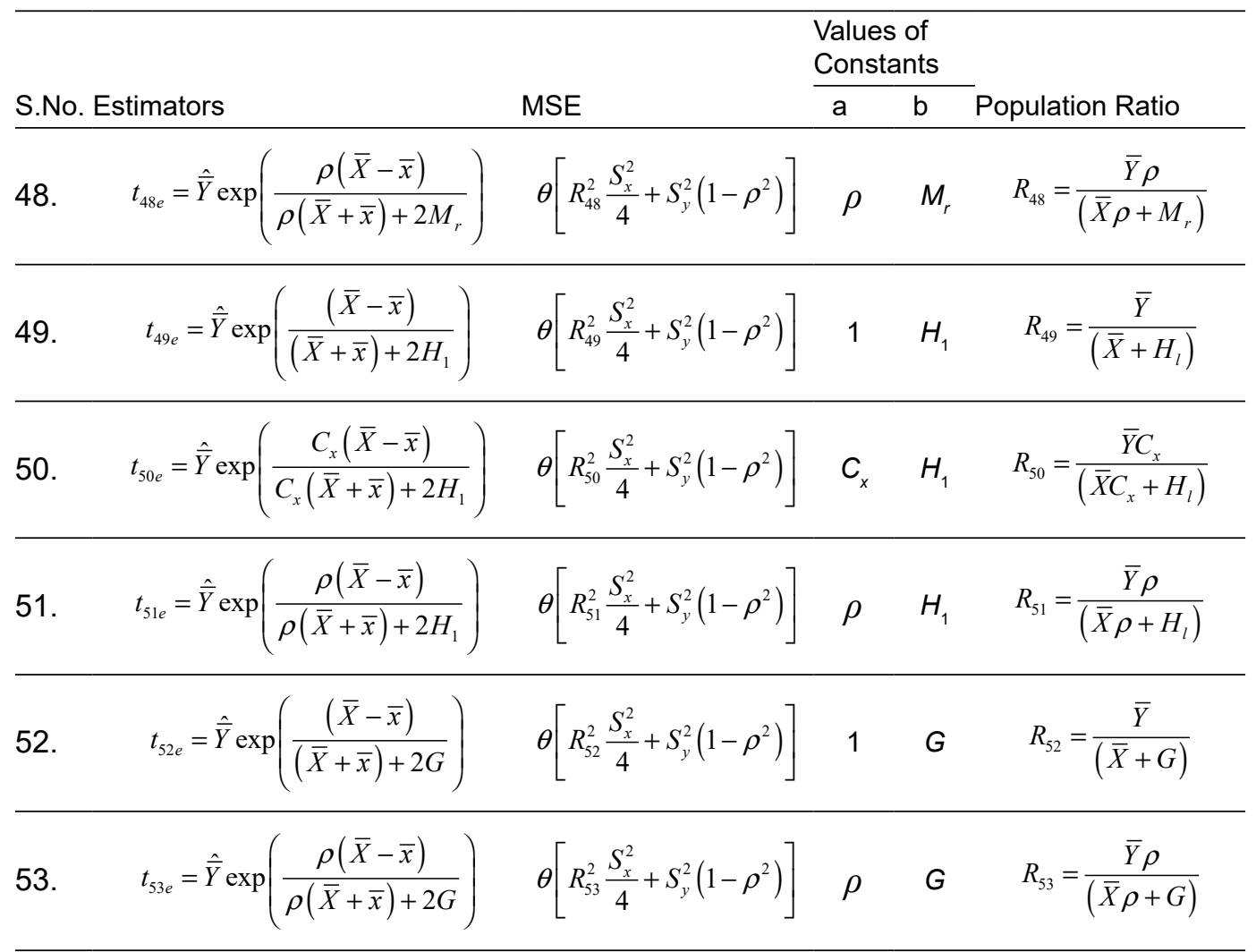

54. $\quad t_{54 e}=\hat{\bar{Y}} \exp \left(\frac{C_{x}(\bar{X}-\bar{x})}{C_{x}(\bar{X}+\bar{x})+2 G}\right) \quad \theta\left[R_{54}^{2} \frac{S_{x}^{2}}{4}+S_{y}^{2}\left(1-\rho^{2}\right)\right] \quad C_{x} \quad G \quad R_{54}=\frac{\bar{Y} C_{x}}{\left(\bar{X} C_{x}+G\right)}$

55. $\quad t_{55 e}=\hat{\bar{Y}} \exp \left(\frac{(\bar{X}-\bar{x})}{(\bar{X}+\bar{x})+2 D}\right) \quad \theta\left[R_{55}^{2} \frac{S_{x}^{2}}{4}+S_{y}^{2}\left(1-\rho^{2}\right)\right] \quad 1 \quad D \quad R_{55}=\frac{\bar{Y}}{(\bar{X}+D)}$

56. $\quad t_{56 e}=\hat{\bar{Y}} \exp \left(\frac{\rho(\bar{X}-\bar{x})}{\rho(\bar{X}+\bar{x})+2 D}\right) \quad \theta\left[R_{56}^{2} \frac{S_{x}^{2}}{4}+S_{y}^{2}\left(1-\rho^{2}\right)\right] \quad \rho \quad D \quad R_{56}=\frac{\bar{Y} \rho}{(\bar{X} \rho+D)}$

57. $\quad t_{57 e}=\hat{\bar{Y}} \exp \left(\frac{C_{x}(\bar{X}-\bar{x})}{C_{x}(\bar{X}+\bar{x})+2 D}\right) \quad \theta\left[R_{57}^{2} \frac{S_{x}^{2}}{4}+S_{y}^{2}\left(1-\rho^{2}\right)\right] \quad C_{x} \quad D \quad R_{57}=\frac{\bar{Y} C_{x}}{\left(\bar{X} C_{x}+D\right)}$

58. $\quad t_{58 e}=\hat{\bar{Y}} \exp \left(\frac{(\bar{X}-\bar{x})}{(\bar{X}+\bar{x})+2 S_{p w}}\right) \quad \theta\left[R_{58}^{2} \frac{S_{x}^{2}}{4}+S_{y}^{2}\left(1-\rho^{2}\right)\right] \quad 1 \quad S_{p w} \quad R_{58}=\frac{\bar{Y} \rho}{\left(\bar{X}+S_{p w}\right)}$

59. $\quad t_{59 e}=\hat{\bar{Y}} \exp \left(\frac{\rho(\bar{X}-\bar{x})}{\rho(\bar{X}+\bar{x})+2 S_{p w}}\right) \quad \theta\left[R_{59}^{2} \frac{S_{x}^{2}}{4}+S_{y}^{2}\left(1-\rho^{2}\right)\right] \quad \rho \quad S_{\rho w} \quad R_{59}=\frac{\bar{Y} \rho}{\left(\bar{X} \rho+S_{p w}\right)}$ 


\section{REDUCING THE MSE OF POPULATION ESTIMATORS}

Table 3. continued

\begin{tabular}{|c|c|c|c|c|c|}
\hline \multirow[b]{2}{*}{ S.No. Estimators } & & \multirow[b]{2}{*}{ MSE } & \multicolumn{2}{|c|}{$\begin{array}{l}\text { Values of } \\
\text { Constants }\end{array}$} & \multirow[b]{2}{*}{ Population Ratio } \\
\hline & & & $a$ & $\mathrm{~b}$ & \\
\hline$t_{60 e}=\hat{\bar{Y}} \exp ($ & $\frac{C_{x}(\bar{X}-\bar{x})}{C_{x}(\bar{X}+\bar{x})+2 S_{p w}}$ & $\theta\left[R_{60}^{2} \frac{S_{x}^{2}}{4}+S_{y}^{2}\left(1-\rho^{2}\right)\right]$ & $C_{x}$ & $S_{p w}$ & $R_{60}=\frac{\bar{Y} C_{x}}{\left(\bar{X} C_{x}+S_{p w}\right)}$ \\
\hline$t_{61 e}=\hat{\bar{Y}} \exp ($ & $\left(\frac{\rho(\bar{X}-\bar{x})}{\rho(\bar{X}+\bar{x})+2 D_{1}}\right.$ & $\theta\left[R_{61}^{2} \frac{S_{x}^{2}}{4}+S_{y}^{2}\left(1-\rho^{2}\right)\right]$ & $\rho$ & $\mathrm{D}_{1}$ & $R_{61}=\frac{\bar{Y} \rho}{\left(\bar{X} \rho+D_{1}\right)}$ \\
\hline$t_{62 e}=\hat{\bar{Y}} \exp ($ & $\left(\frac{\rho(\bar{X}-\bar{x})}{\rho(\bar{X}+\bar{x})+2 D_{2}}\right.$ & $\theta\left[R_{62}^{2} \frac{S_{x}^{2}}{4}+S_{y}^{2}\left(1-\rho^{2}\right)\right]$ & $\rho$ & $D_{2}$ & $R_{62}=\frac{\bar{Y} \rho}{\left(\bar{X} \rho+D_{2}\right)}$ \\
\hline$t_{63 e}=\hat{\bar{Y}} \exp$ & $\left(\frac{\rho(\bar{X}-\bar{x})}{\rho(\bar{X}+\bar{x})+2 D_{3}}\right.$ & $\theta\left[R_{63}^{2} \frac{S_{x}^{2}}{4}+S_{y}^{2}\left(1-\rho^{2}\right)\right]$ & $\rho$ & $\mathrm{D}_{3}$ & $R_{63}=\frac{\bar{Y} \rho}{\left(\bar{X} \rho+D_{3}\right)}$ \\
\hline$t_{64 e}=\hat{\bar{Y}} \exp ($ & $\left(\frac{\rho(\bar{X}-\bar{x})}{\rho(\bar{X}+\bar{x})+2 D_{4}}\right.$ & $\theta\left[R_{64}^{2} \frac{S_{x}^{2}}{4}+S_{y}^{2}\left(1-\rho^{2}\right)\right]$ & $\rho$ & $\mathrm{D}_{4}$ & $R_{64}=\frac{\bar{Y} \rho}{\left(\bar{X} \rho+D_{4}\right)}$ \\
\hline$t_{65 e}=\hat{\bar{Y}} \exp ($ & $\frac{\rho(\bar{X}-\bar{x})}{\rho(\bar{X}+\bar{x})+2 D_{5}}$ & $\theta\left[R_{65}^{2} \frac{S_{x}^{2}}{4}+S_{y}^{2}\left(1-\rho^{2}\right)\right]$ & $\rho$ & $\mathrm{D}_{5}$ & $R_{65}=\frac{\bar{Y} \rho}{\left(\bar{X} \rho+D_{5}\right)}$ \\
\hline$t_{66 e}=\hat{\bar{Y}} \exp$ & $\frac{\rho(\bar{X}-\bar{x})}{\rho(\bar{X}+\bar{x})+2 D_{6}}$ & $\theta\left[R_{66}^{2} \frac{S_{x}^{2}}{4}+S_{y}^{2}\left(1-\rho^{2}\right)\right]$ & $\rho$ & $\mathrm{D}_{6}$ & $R_{66}=\frac{\bar{Y} \rho}{\left(\bar{X} \rho+D_{6}\right.}$ \\
\hline$t_{67 e}=\hat{\bar{Y}} \exp$ & $\frac{\rho(\bar{X}-\bar{x})}{\rho(\bar{X}+\bar{x})+2 D_{7}}$ & $\theta\left[R_{67}^{2} \frac{S_{x}^{2}}{4}+S_{y}^{2}\left(1-\rho^{2}\right)\right]$ & $\rho$ & $\mathrm{D}_{7}$ & $R_{67}=\frac{\bar{Y} \rho}{\left(\bar{X} \rho+D_{7}\right)}$ \\
\hline$t_{68 e}=\hat{\bar{Y}} \exp$ & $\frac{\rho(\bar{X}-\bar{x})}{\rho(\bar{X}+\bar{x})+2 D_{8}}$ & $\theta\left[R_{68}^{2} \frac{S_{x}^{2}}{4}+S_{y}^{2}\left(1-\rho^{2}\right)\right]$ & $\rho$ & $\mathrm{D}_{8}$ & $R_{68}=\frac{\bar{Y} \rho}{\left(\bar{X} \rho+D_{8}\right)}$ \\
\hline$t_{69 e}=\hat{\bar{Y}} \exp$ & $\left(\frac{\rho(\bar{X}-\bar{x})}{\rho(\bar{X}+\bar{x})+2 D_{9}}\right.$ & $\theta\left[R_{69}^{2} \frac{S_{x}^{2}}{4}+S_{y}^{2}\left(1-\rho^{2}\right)\right]$ & $\rho$ & $\mathrm{D}_{9}$ & $R_{69}=\frac{\bar{Y} \rho}{\left(\bar{X} \rho+D_{9}\right)}$ \\
\hline$t_{70 e}=\hat{\bar{Y}} \exp$ & $\left(\frac{\rho(\bar{X}-\bar{x})}{\rho(\bar{X}+\bar{x})+2 D_{10}}\right.$ & $\theta\left[R_{70}^{2} \frac{S_{x}^{2}}{4}+S_{y}^{2}\left(1-\rho^{2}\right)\right]$ & $\rho$ & $\mathrm{D}_{10}$ & $R_{70}=\frac{\bar{Y} \rho}{\left(\bar{X} \rho+D_{10}\right)}$ \\
\hline
\end{tabular}


HOUSILA P. SINGH \& ANITA YADAV

Table 3. continued

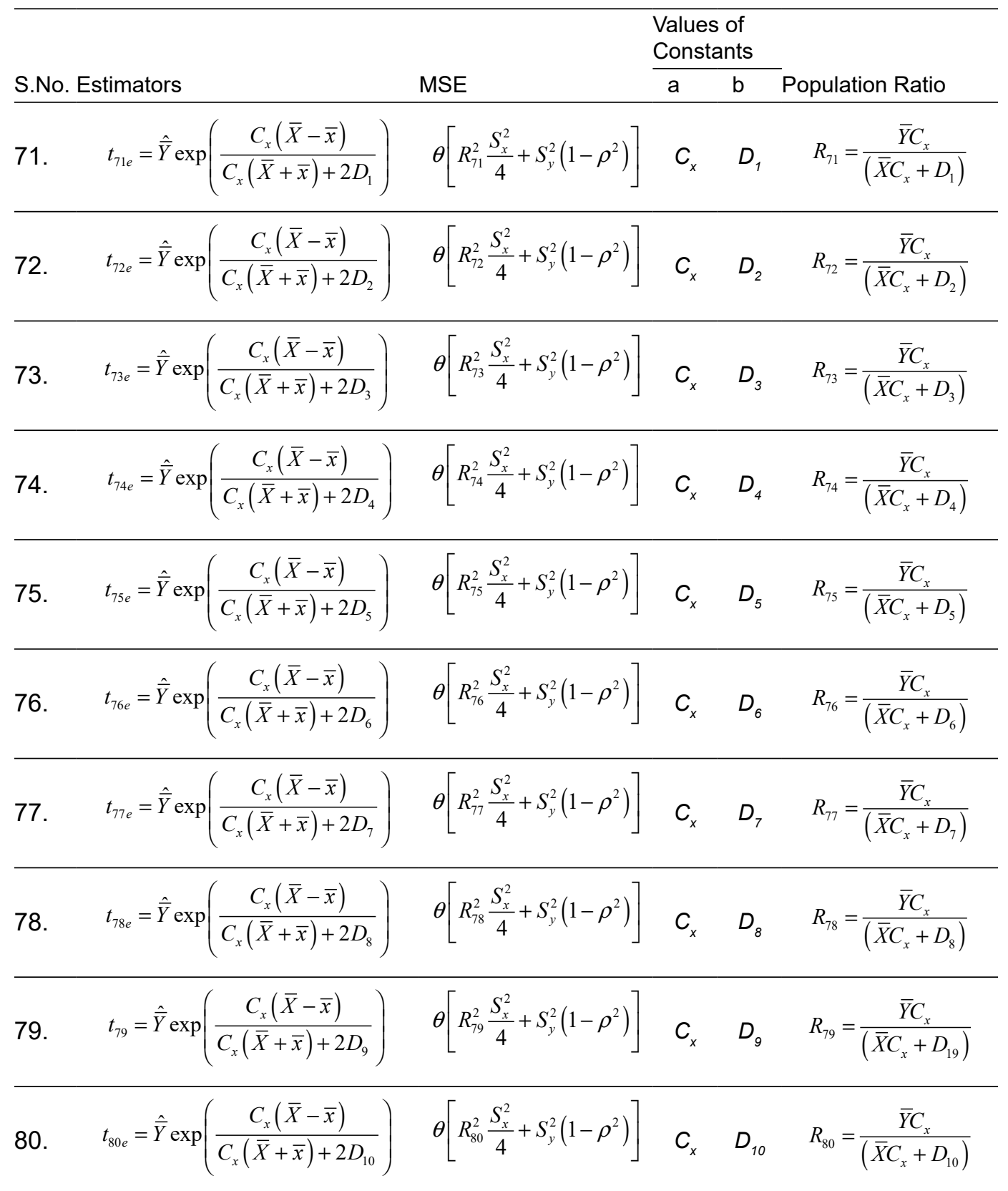




\section{REDUCING THE MSE OF POPULATION ESTIMATORS}

The bias of $t_{e}$ at (17) is negligible if the sample size $\mathrm{n}$ is sufficiently large. The bias of the members of the proposed class of estimators can be obtained easily from (17) just by putting suitable values of the scalars $(a, b)$. Squaring both sides of (16) and neglecting terms of e's having power greater than two

$$
\left(t_{e}-\bar{Y}\right)^{2}=\bar{Y}^{2}\left[e_{0}^{2}+\frac{\tau^{2} e_{1}^{2}}{4}+C^{2} e_{1}^{2}-\tau e_{0} e_{1}-2 C e_{0} e+\tau C e_{1}^{2}\right]
$$

Taking expectation of both sides of (18) we get the MSE of $t_{e}$ to the first degree of approximation as

$$
\operatorname{MSE}\left(t_{e}\right)=\frac{(1-f)}{n}\left[R_{J}^{2}\left(\frac{S_{x}^{2}}{4}\right)+S_{y}^{2}\left(1-\rho^{2}\right)\right] .
$$

The MSE of the members of the proposed class of estimators $t_{e}$ can be easily obtained from (19) just by putting the suitable values of $(a, b)$.

Remark 1 Motivated by Swain (2014), define a class of ratio-type estimators for population mean $\bar{Y}$ as

$$
t_{s}=\hat{\bar{Y}}\left(\frac{a \bar{X}+b}{a \bar{x}+b}\right)^{1 / 2} .
$$

Thus the form of the estimators $t$ and $t_{S}$ taking into consideration, we define a class of ratio-cum-product-type estimators for population mean $\bar{Y}$ as

$$
t_{g}=\hat{\bar{Y}}\left(\frac{a \bar{X}+b}{a \bar{x}+b}\right)^{g}
$$

where $g$ is a scalar taking real values. Note for $g(>0)$ the class of estimators $t_{g}$ generates the ratio-type estimators while for $\mathrm{g}(<0)$ it generates product-type estimators.

To the first degree of approximation the bias and MSE of $t_{e}$ are respectively given by

$$
\begin{gathered}
B\left(t_{g}\right)=\frac{(1-f)}{n}\left[R_{J}^{2} \frac{S_{x}^{2}}{\bar{Y}} \frac{g(g+1)}{2}-\frac{\beta N}{(N-2)}\left(\frac{\mu_{21}}{\mu_{11}}-\frac{\mu_{30}}{\mu_{20}}\right)\right] \\
=\frac{(1-f)}{n}\left[\frac{g(g+1)}{2} A-B\right],
\end{gathered}
$$




$$
\operatorname{MSE}\left(t_{g}\right)=\frac{(1-f)}{n}\left[g^{2} R_{J}^{2} S_{x}^{2}+S_{y}^{2}\left(1-\rho^{2}\right)\right]
$$

where $A$ and $B$ are same as defined earlier. Putting $g=\frac{1}{2}$ in (22) and (23) we get the bias and MSE of the Swain's (2014)-type estimator $t_{S}$ at (20) respectively as

$$
B\left(t_{s}\right)=\frac{(1-f)}{n}\left[\frac{3}{8} A-B\right]
$$

and

$$
\operatorname{MSE}\left(t_{s}\right)=\frac{(1-f)}{n}\left[\frac{R_{J}^{2} S_{x}^{2}}{4}+S_{y}^{2}\left(1-\rho^{2}\right)\right] .
$$

From (17), (19), (24) and (25), note the bias and MSE of the Swain's (2014) type estimators $t_{S}$ and ratio-type exponential estimator $t_{e}$ defined in (14) are same up to first order of approximation.

From (11) and (23)

$$
\operatorname{MSE}(t)-\operatorname{MSE}\left(t_{g}\right)=\frac{(1-f)}{n} R_{J}^{2} S_{x}^{2}+S_{x}^{2}\left(1-g^{2}\right)
$$

which is positive if

$$
\begin{array}{cc} 
& 1-g^{2}>0 \\
\text { i.e if } & g^{2}<1 \\
\text { i.e if } & -1<g<1 .
\end{array}
$$

The members of the class of ratio-cum-product type estimators $t_{g}$ is more efficient than the corresponding members of the proposed class of ratio-type estimators $t$ as long as the condition (26) is satisfied. From (19) and (23)

$$
\operatorname{MSE}(t)-\operatorname{MSE}\left(t_{g}\right)=\frac{(1-f)}{n} R_{J}^{2} S_{x}^{2}+\left(\frac{1}{4}-g^{2}\right)
$$

which is positive if

i.e. if

$$
\left(\frac{1}{4}-g^{2}\right)>0 g^{2}>\frac{1}{4}
$$




\section{REDUCING THE MSE OF POPULATION ESTIMATORS}

i.e. if $\quad-\frac{1}{2}<g<\frac{1}{2}$.

The proposed class of ratio-cum-product type estimators $t_{g}$ would always be more efficient than the corresponding members of the ratio-type exponential estimator $t_{e}$ as long as the condition (27) is satisfied.

Remark 2 It follows from (23) that either the estimator is ratio-type (i.e. $t$ defined by (6) ) or product-type defined by

$$
\begin{gathered}
t^{* *}=\hat{\bar{Y}}\left(\frac{a \bar{x}+b}{a \bar{X}+b}\right), \\
=[\bar{y}+\hat{\beta}(\bar{X}-\bar{x})]\left(\frac{a \bar{x}+b}{a \bar{X}+b}\right),
\end{gathered}
$$

the mean squared errors of ratio-type $(t)$ and product-type $\left(t^{* *}\right)$ to the first degree of approximation are turn out to be the same i.e.

$$
\operatorname{MSE}(t)=\operatorname{MSE}\left(t^{* *}\right)=\frac{(1-f)}{n}\left[R_{J}^{2} S_{x}^{2}+S_{y}^{2}\left(1-\rho^{2}\right)\right] .
$$

The proposed class of estimators is always better than the ratio-type $(t)$ and product-type $\left(t^{* *}\right)$ estimators as long as the condition:

$$
-1<g<1
$$

is satisfied.

Remark 3 Define a generalized version of the ratio-cum-product-type exponential estimator $t_{e}$ for the population mean $\bar{Y}$ as

$$
\begin{gathered}
t_{g e}=\hat{\bar{Y}} \exp \left\{\frac{g\left(\bar{X}^{*}-\bar{x} *\right)}{\left(\bar{X}^{*}+\bar{x} *\right)}\right\} \\
=[\bar{y}+\hat{\beta}(\bar{X}-\bar{x})] \exp \left\{\frac{g a(\bar{X}-\bar{x})}{(\bar{X}+\bar{x})+2 b}\right\},
\end{gathered}
$$


where $\bar{x}^{*}=(a \bar{x}+b)$ such that $E\left(\bar{x}^{*}\right)=\bar{X}^{*}=a \bar{X}+b$; and $g$ is a scalar taking real values. For $g(>0) t_{g e}$ generates ratio-type exponential estimator, and for $g(>0)$ it generates product-type exponential estimator.

To the first degree of approximation, the bias and mean squared error of the proposed class of ratio-cum-product-type exponential estimators $t_{g e}$ are respectively given by

$$
\begin{gathered}
B\left(t_{g e}\right)=\frac{(1-f)}{n}\left[\frac{g(g+2)}{8} R_{J}^{2} \frac{S_{x}^{2}}{\bar{Y}}-\frac{N}{(N-2)} \beta\left(\frac{\mu_{21}}{\mu_{11}}-\frac{\mu_{30}}{\mu_{20}}\right)\right] \\
=\frac{(1-f)}{n}\left[\frac{g(g+2)}{8} A-B\right]
\end{gathered}
$$

and

$$
\operatorname{MSE}\left(t_{g e}\right)=\frac{(1-f)}{n}\left[\left(\frac{g^{2}}{4}\right) R_{J}^{2} S_{x}^{2}+S_{y}^{2}\left(1-\rho^{2}\right)\right] .
$$

From (23) and (33)

$$
\operatorname{MSE}\left(t_{g}\right)-\operatorname{MSE}\left(t_{g e}\right)=\frac{3}{4} \frac{(1-f)}{n} g^{2} R_{J}^{2} S_{x}^{2}
$$

which is always positive.

It follows from (34) that the members of the proposed class of ratio-cum-producttype exponential estimators $t_{g e}$ is always better than the corresponding members of the suggested class of ratio-cum-product type estimators $t_{e}$.

From (19) and (33) we have

$$
\operatorname{MSE}\left(t_{e}\right)-\operatorname{MSE}\left(t_{g e}\right)=\frac{(1-f)}{n} R_{J}^{2} \frac{S_{x}^{2}}{4}\left(1-g^{2}\right)
$$

which is positive if

$$
\left(1-g^{2}\right)>0
$$

i.e. if

$$
-1<g<1
$$

i.e. if

$$
|g|<1 \text {. }
$$


The members of the proposed class of ratio-cum product-type exponential estimators is better than the corresponding members of the suggested ratio-type exponential estimator ${ }^{t_{e}}$ as long as the condition (35) is satisfied. It can be also proved that the members proposed of the proposed class of ratio-cum-product-type estimators $t_{g e}$ is also better than the corresponding members of the product-type exponential estimator defined by

$$
\begin{gathered}
t_{e}^{* *}=\hat{\bar{Y}} \exp \left\{\frac{a(\bar{x}-\bar{X})}{a(\bar{X}+\bar{x})+2 b}\right\} \\
=\{\bar{y}+\hat{\beta}(\bar{X}-\bar{x})\} \exp \left\{\frac{a(\bar{x}-\bar{X})}{a(\bar{X}+\bar{x})+2 b}\right\}
\end{gathered}
$$

as long as the condition: $|g|<1$ in (35) is satisfied.

\section{Efficiency Comparison}

From (2) and (19)

$$
\operatorname{MSE}(\bar{y})-\operatorname{MSE}\left(t_{e}\right)=\frac{(1-f)}{n} S_{x}^{2}\left(\beta^{2}-\frac{R_{j}^{2}}{4}\right)
$$

which is positive if

$$
\beta^{2}-\frac{R_{J}^{2}}{4}>0
$$

i.e. if

$$
\frac{R_{J}^{2}}{4}<\beta^{2} \text {. }
$$

From (5) and (19) e

$$
\operatorname{MSE}\left(\bar{y}_{R}\right)-\operatorname{MSE}\left(t_{e}\right)=\frac{(1-f)}{n}\left[(\beta-R)^{2}-\frac{R_{J}^{2}}{4}\right]
$$

which is non-negative if

$$
\left[(\beta-R)^{2}-\frac{R_{j}^{2}}{4}\right]>0
$$


i.e. if

$$
\frac{R_{j}^{2}}{4}<(\beta-R)^{2}
$$

Further from (11) and (19) we have

$$
\operatorname{MSE}(t)-\operatorname{MSE}\left(t_{e}\right)=\frac{3}{4} \frac{(1-f)}{n} R_{J}^{2} S_{x}^{2}
$$

which is always positive.

Thus from (37) - (39) it follows that the members of the proposed class of estimators $t_{e}$ is :

(i) more efficient than the usual unbiased estimator $\bar{y}$ as long as the condition (37) is satisfied.

(ii) more efficient than the usual ratio estimator $\bar{y}_{R}$ as long as the condition (38) is satisfied.

(iii) is always better than the corresponding members of the $t$-family of estimators.

\section{Bias Comparison the Estimators $t$ and $t_{e}$}

It follows from (9) and (17)

$$
\left|B\left(t_{e}\right)\right|<|B(t)|
$$

if

$$
\left|\frac{3}{8} A-B\right|<|A-B|
$$

Since

$$
\left|\frac{3}{8} A-B\right|<\frac{3}{8}|A|+|B|
$$

and

$$
|A-B|<\backslash|A|+|B| .
$$




\section{REDUCING THE MSE OF POPULATION ESTIMATORS}

Therefore, from (40), (41) and (42)

$$
\left|B\left(t_{e}\right)\right|<|B(t)|
$$

if

$$
\frac{3}{8}|A|+|B|<|A|+|B|
$$

i.e. if $\quad \frac{3}{8}|A|<|A|$

i.e. if $\frac{5}{8}|A|>0$

which is always true. The members of the proposed $t_{e}$ - family of estimators are less biased as well as more efficient than the corresponding members of the $t$ - family. Hence, the members of the proposed class of estimators $t_{e}$ is more efficient than the corresponding known members due to Kadilar and Cingi (2004), Kadilar and Cingi (2006)- type, Yan and Tian (2010), Subramani and Kumarapandian (2012a, 2012b, 2012c, 2012d), Jeelani et al (2013) and Abid et al (2016a, 2016b, 2016c) of the class of estimators $t$.

\section{Empirical Study}

In support of the theoretical results, MSEs of some known estimators listed in Table 1 were computed, and corresponding estimators listed in Table 3. Natural data sets were those considered by Kadilar and Cingi (2004) and Abid et al (2012b). The findings are shown in Table 4.

Population- Source: Kadilar and Cingi (2004) and Abid et al (2016b, p.361).

$y$ : Apple production

$x$ : Number of apple trees

$$
\begin{array}{llll}
N=106, & n=40, & \bar{Y}=2212.59, & \bar{X}=27421.70 \\
\rho=0.860, & S_{y}=11551.53, & C_{y}=5.22, & S_{x}=57460.61, \\
C_{x}=2.10 & \beta_{2}(x)=34.572, & \beta_{1}(x)=2.122, & M_{d}=7297.50 \\
Q_{d}=12156.25, & G=40201.69 & S_{p w}=35298.810, & D=35634.990
\end{array}
$$




\section{HOUSILA P. SINGH \& ANITA YADAV}

Table 4. Mean Squared Errors of some known members of the class of ratio-cum-product estimators $t$ and the corresponding members of the class of ratio-cum-product-type estimators $t_{\mathrm{e}}$

\begin{tabular}{|c|c|c|c|c|c|}
\hline Known Estimators & $\operatorname{MSE}(t)$ & Rank & Corresponding members of $t_{e}$ & $\operatorname{MSE}\left(t_{e}\right)$ & Rank \\
\hline $\begin{array}{l}t_{1}=\frac{\hat{\bar{Y}}}{\bar{x}} \cdot \bar{X} \\
\text { Kadilar and Cingi (2004) }\end{array}$ & 875480.89 & $X X V I$ & $t_{1 e}=\hat{\bar{Y}} \exp \left(\frac{\bar{X}-\bar{x}}{\bar{X}+\bar{x}}\right)$ & 624527.57 & $X X V I$ \\
\hline$t_{2}=\frac{\hat{\bar{Y}}}{\left(\bar{x}+C_{x}\right)}\left(\bar{X}+C_{x}\right)$ & 875429.64 & $X X I$ & $t_{2 e}=\hat{\bar{Y}} \exp \left(\frac{\bar{X}-\bar{x}}{\bar{X}+\bar{x}+2 C_{x}}\right)$ & 624514.75 & $X X I$ \\
\hline
\end{tabular}

Kadilar and Cingi (2004)

$t_{3}=\frac{\hat{\bar{Y}}}{\left(\bar{x}+\beta_{2}(x)\right)}\left(\bar{X}+\beta_{2}(x)\right) \quad 874638.77 \quad x V I \quad t_{3 e}=\hat{\bar{Y}} \exp \left(\frac{(\bar{X}-\bar{x})}{\bar{X}+\bar{x}+2 \beta_{2}(x)}\right) \quad 624317.04 \quad X V I$ Kadilar and Cingi (2004)

$t_{4}=\frac{\hat{\bar{Y}}}{\left(\bar{x} \beta_{2}(x)+C_{x}\right)}\left(\bar{X} \beta_{2}(x)+C_{x}\right) \quad 875479.40 \quad$ XXIV $\quad t_{4 e}=\hat{\bar{Y}} \exp \left(\frac{(\bar{X}-\bar{x}) \beta_{2}(x)}{\beta_{2}(x)(\bar{X}+\bar{x})+2 C_{x}}\right) \quad 624527.20 \quad$ XXIV Kadilar and Cingi (2004)

$t_{5}=\frac{\hat{\bar{Y}}}{\left(\bar{x} C_{x}+\beta_{2}(x)\right)}\left(\bar{X} C_{x}+\beta_{2}(x)\right) \quad 875079.48 \quad X V I I \quad t_{5 e}=\hat{\bar{Y}} \exp \left(\frac{C_{x}(\bar{X}-\bar{x})}{C_{x}(\bar{X}+\bar{x})+2 \beta_{2}(x)}\right) \quad 624427.21 \quad$ XVII
Kadilar and Cingi (2004)

$t_{6}=\frac{\hat{\bar{Y}}}{(\bar{x}+\rho)}(\bar{X}+\rho)$
Kadilar and Cingi (2006) -type

$t_{7}=\frac{\hat{\bar{Y}}}{\left(\bar{x} C_{x}+\rho\right)}\left(\bar{X} C_{x}+\rho\right)$
(2006 Kadilar and Cingi) -type

$t_{8}=\frac{\hat{\bar{Y}}}{\left(\bar{x} \rho+C_{x}\right)}\left(\bar{X} \rho+C_{x}\right)$
Kadilar and Cingi (2006) -type

$t_{9}=\frac{\hat{\bar{Y}}}{\left(\bar{x} \beta_{2}(x)+\rho\right)}\left(\bar{X} \beta_{2}(x)+\rho\right) \quad 875480.28 \quad X X V \quad t_{9 e}=\hat{\bar{Y}} \exp \left(\frac{\beta_{2}(x)(\bar{X}-\bar{x})}{\beta_{2}(x)(\bar{X}+\bar{x})+2 \rho}\right) \quad 624527.41 \quad x X V$

Kadilar and E Cingi (2006) -type

$\begin{aligned} & t_{10}=\frac{Y}{\left(\bar{x} \rho+\beta_{2}(x)\right)}\left(\bar{X} \rho+\beta_{2}(x)\right) \\ & \text { Kadilar and Cingi (2006) -type }\end{aligned} \quad 874501.98 \quad x V \quad t_{10 e}=\hat{\bar{Y}} \exp \left(\frac{\rho(\bar{X}-\bar{x})}{\rho(\bar{X}+\bar{x})+2 \beta_{2}(x)}\right) \quad 624282.84 \quad x V$

$t_{11}=\frac{\hat{\bar{Y}}}{\left(\bar{x}+\beta_{1}(x)\right)}\left(\bar{X}+\beta_{1}(x)\right)$

875429.11 $X X \quad t_{11 e}=\hat{\bar{Y}} \exp \left(\frac{(\bar{X}-\bar{x})}{(\bar{X}+\bar{x})+2 \beta_{1}(x)}\right) \quad 624514.62 \quad x X$

Yan and Tian (2010)

continued 


\section{REDUCING THE MSE OF POPULATION ESTIMATORS}

Table 4. continued

\begin{tabular}{|c|c|c|c|c|c|}
\hline Known Estimators & $\operatorname{MSE}(t)$ & Rank & Corresponding members of $t_{e}$ & $\operatorname{MSE}\left(t_{e}\right)$ & Rank \\
\hline $\begin{array}{l}t_{12}=\frac{\hat{\bar{Y}}}{\left(\bar{x} \beta_{1}(x)+\beta_{2}(x)\right)}\left(\bar{X} \beta_{1}(x)+\beta_{2}(x)\right) \\
\text { Yan and Tian (2010) }\end{array}$ & 875083.64 & XVIII & $t_{12 e}=\hat{\bar{Y}} \exp \left(\frac{\beta_{1}(x)(\bar{X}-\bar{x})}{\beta_{1}(x)(\bar{X}+\bar{x})+2 \beta_{2}(x)}\right.$ & 624428.25 & $X V I I I$ \\
\hline $\begin{array}{l}t_{13}=\frac{\hat{\bar{Y}}}{\left(\bar{x}+M_{d}\right)}\left(\bar{X}+M_{d}\right) \\
\text { Subramani and Kumarapandian (2012a) }\end{array}$ & 749604.60 & $x$ & $t_{13 e}=\hat{\bar{Y}} \exp \left(\frac{(\bar{X}-\bar{x})}{(\bar{X}+\bar{x})+2 M_{d}}\right.$ & 593058.50 & $x$ \\
\hline $\begin{array}{l}t_{14}=\frac{\hat{\bar{Y}}}{\left(C_{x} \bar{x}+M_{d}\right)}\left(\bar{X} C_{x}+M_{d}\right) \\
\text { Subramani and Kumarapandian (2012a) }\end{array}$ & 804446.62 & $X I I$ & $t_{14 e}=\hat{\bar{Y}} \exp \left(\frac{C_{x}(\bar{X}-\bar{x})}{C_{x}(\bar{X}+\bar{x})+2 M_{d}}\right.$ & 606769.00 & $X I I$ \\
\hline
\end{tabular}

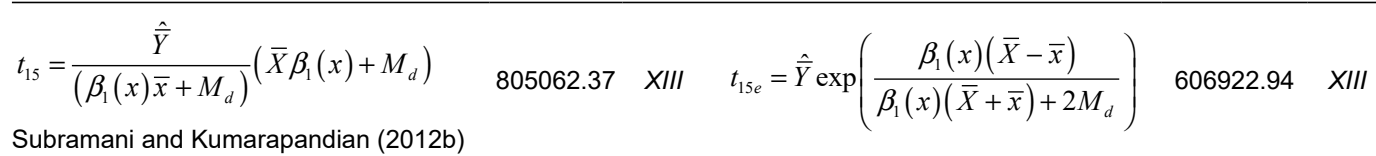

$t_{16}=\frac{\hat{\bar{Y}}}{\left(\beta_{2}(x) \bar{x}+M_{d}\right)}\left(\bar{X} \beta_{2}(x)+M_{d}\right) \quad 870388.46 \quad X V \quad t_{16 e}=\hat{\bar{Y}} \exp \left(\frac{\beta_{2}(x)(\bar{X}-\bar{x})}{\beta_{2}(x)(\bar{X}+\bar{x})+2 M_{d}}\right) \quad 623254.46 \quad X I V$

Subramani and Kumarapandian (2012c)

$t_{27}=\frac{\hat{\bar{Y}}}{\left(\bar{x} \beta_{1}(x)+Q_{d}\right)}\left(\bar{X} \beta_{1}(x)+Q_{d}\right) \quad 769827.97 \quad X I \quad t_{27 e}=\hat{\bar{Y}} \exp \left(\frac{\beta_{1}(x)(\bar{X}-\bar{x})}{\beta_{1}(x)(\bar{X}+\bar{x})+2 Q_{d}}\right) \quad 598114.34 \quad X I$
Jeelani et al (2013)

$t_{37}=\frac{\hat{\bar{Y}}}{(\bar{x}+G)}(\bar{X}+G) \quad 595897.22 \quad I V \quad t_{37 e}=\hat{\bar{Y}} \exp \left(\frac{(\bar{X}-\bar{x})}{(\bar{X}+\bar{x})+2 G}\right) \quad 554631.65 \quad I V$
Abid et al (2016b)

Abid et al (2016b)

$t_{38}=\frac{\hat{\bar{Y}}}{(\bar{x} \rho+G)}(\bar{X} \rho+G)$

$$
t_{38 e}=\hat{\bar{Y}} \exp \left(\frac{\rho(\bar{X}-\bar{x})}{\rho(\bar{X}+\bar{x})+2 G}\right)
$$

$552311.27 \quad$

Abid et al (2016b)

$t_{39}=\frac{\hat{\bar{Y}}}{\left(\bar{x} C_{x}+G\right)}\left(\bar{X} C_{x}+G\right) \quad 656912.92 \quad$ VII $\quad t_{39 e}=\hat{\bar{Y}} \exp \left(\frac{C_{x}(\bar{X}-\bar{x})}{C_{x}(\bar{X}+\bar{x})+2 G}\right) \quad 569885.57 \quad$ VII

Abid et al (2016b)

$t_{40}=\frac{\hat{\bar{Y}}}{(\bar{x}+D)}(\bar{X}+D)$

$604155.24 \quad V \quad t_{40 e}=\hat{\bar{Y}} \exp \left(\frac{(\bar{X}-\bar{x})}{(\bar{X}+\bar{x})+2 D}\right)$

$556696.15 \quad V$

Abid et al (2016b)

$t_{41}=\frac{\hat{\bar{Y}}}{(\bar{x} \rho+D)}(\bar{X} \rho+D)$
Abid et al (2016b)


HOUSILA P. SINGH \& ANITA YADAV

Table 4. continued

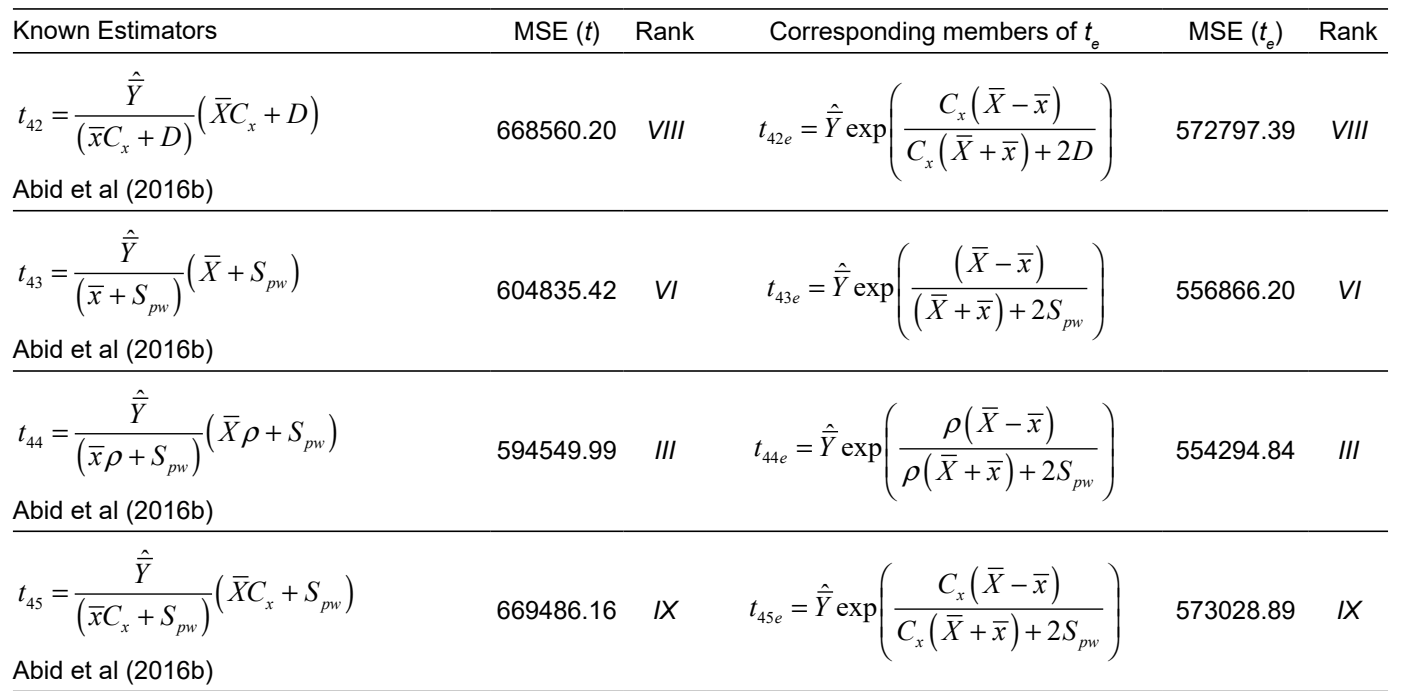

It is observed from Table 4 there is considerable reduction in MSEs of the proposed estimators $\left(t_{1 e}\right.$ to $t_{16 e}, t_{27 e}, t_{37 e}$ to $\left.t_{45 e}\right)$ as compared to the corresponding known estimators $\left(t_{1}\right.$ to $t_{13}, t_{27}, t_{37}$ to $\left.t_{45}\right)$. That is the members of the proposed class of ratiocum-product-type exponential estimators $t_{e}$ is more efficient than the corresponding members of the class of ratio-cum-product-type estimators $t$. The proposed estimators $t_{38 e}$ followed by the estimator $t_{41 e}$ have the smallest MSE among all the estimators considered in Table 4. Thus, the proposed class of ratio-cum-product-type exponential estimators $t_{e}$ is justified.

\section{References}

Abid, M., Abbas, N. \& Riaz, M. (2016a). Enhancing the mean ratio estimators for estimating population mean using non-conventional location parameters, Revista Colombiana de Estadistica, 39(1), 63-79. doi: 10.15446/rce.v39n1.55139

Abid, M., Abbas, N. \& Riaz, M. (2016b). Improved Modified Ratio Estimators of population mean based on deciles, Chiang Mai Journal of Science, 43(1), 1311-1323.

Abid, M., Abbas, N., Sherwani, K. A. R, \& Nazir, Z. H. (2016c). Improved ratio estimators for the population mean using non-conventional measures of dispersion, Pakistan Journal of Statistics and Operation Research, 12(2), 353-367. doi: 10.18187/pjsor.v12i2.1182

Al-Omari, A. I., Jemain, A. A. \& Ibrahim, K. (2009). New ratio estimators of the mean using simple random sampling and ranked set sampling methods, Revsta Investigacion Operacional, 30(2), 97-108. 


\section{REDUCING THE MSE OF POPULATION ESTIMATORS}

Das A. K. \& Tripathi. T. P. (1980). Sampling strategies for population mean when two coefficient of variation of an auxiliary character is known. Shankya, C, 42(12), 78-60.

Ekpenyoung, E. J. \& Enang, E. I. (2015). A Modified Class of Ratio and Product Estimators of the Population Mean in Simple Random Sampling using Information on Auxiliary Variable. Journal of Statistics, 22, 1-8.

Jeelani, M. I., Maqbool, S. \& Mir, S. A. (2013). Modified ratio estimators of population mean using linear combination of coefficient of skewness and quartile deviation. International Journal of Modern Mathematical Sciences (6), 174-183.

Kadilar, C. \& Cingi, H. (2004). Ratio estimators in simple random sampling. Applied Mathematics and Computaton, 151(3), 893-902. doi: 10.1016/s0096-3003(03)00803-8

Kadilar, C. \& Cingi, H. (2006). An improvement in estimating the population mean by using the correlation coefficient, Hacettepe Journal of Mathematics and Statistics, 35(1), 103-109.

Kumarapandiyan, G. \& Subramani, J. (2016). Some modified ratio estimators for estimation of population mean using known parameters of an auxiliary variable, Statistical Modelling and Analysis Techniques, 93-101.

Searls, D. T. (1964). The Utilization of a known coefficient of variation in the estimation procedure. Journal of American Statistical Association., 59(308), 1225-1226. doi: 10.1080/01621459.1964.1048076

Swain, A. K. P. C. (2014). An Improved ratio type estimator of finite population in sample surveys, Revsta Investigacion Operacional, 35(1), 49-57.

Singh, G. N. (2003). On the improvement of product method of estimation in sample surveys, Journal of the Indian Society of Agricultural Statistics, 56(3), 267-275.

Singh, H. P. \& Tailor, R. (2003). Use of known correlation coefficient in estimating the finite population means, Statistics in Transition, 6(4), 555-560.

Singh, H. P., Tailor, R., Tailor, R. and Kakran, M. S. (2004). An improved estimator of population mean using power transformation. Journal of the Indian Society of Agricultural Statistics, (58), 223-230.

Sisodia, B. V. S. \& Dwivedi, V. K. (1981). A modified ratio estimator using coefficient of variation of auxiliary varianle. Journal of the Indian Society of Agricultural Statistics, 33(1), 13-18.

Subramani, J. \& Kumarapandiyan, G. (2012a). Estimation of Population Mean using Coefficient of variation and Median of an Auxiliary Variable. International Journal of Probability and Statistics, 1(4), 111-118.

Subramani, J. \& Kumarapandiyan, G. (2012b). Estimation of population mean using known median and Co-efficient of skewness. American Journal of Mathematics and Statistics, 2(5), 101-107. doi: 10.5923/j.ajms.20120205.01

Subramani, J. \& Kumarapandiyan, G. (2012c). Modified ratio estimators using known median and co-efficient of kurtosis. American Journal of Mathematics and Statistics, 2(4), 95-100. doi: 10.5923/j.ajms.20120204.05

Subramani, J. \& Kumarapandiyan, G. (2012d). A class of modified ratio estimators using Deciles of an Auxiliary Variable. International Journal of Statistics and Applications, 2(6), 101-107. doi: 10.5923/j.statistics.20120206.02 


\section{HOUSILA P. SINGH \& ANITA YADAV}

Subramani, J., Kumarapandiyan, G., \& Balamurali, S. (2015). A Class of Modified Linear Regression Type Ratio Estimators of Population Mean using Coefficent of Variation and Quartiles of an Auxiliary Variable. Journal of Statistics, 22, 284-305

Upadhyaya, L. N. \& Singh, H. P. (1999). Use of transformed auxiliary variable in estimating the finite population mean. Biometrical Journal, 41(5), 627-636. doi: 10.1002/ (sici)1521-4036(199909)41:5<627::aid-bimj627>3.0.co;2-w

Yan Z., Tian B. (2010). Ratio Method to the Mean Estimation Using Coefficient of Skewness of Auxiliary Variable. In: Zhu R., Zhang Y., Liu B., Liu C. (Eds.). Information Computing and Applications. ICICA 2010. Communications in Computer and Information Science, vol 106. Springer, Berlin, Heidelberg. doi: 10.1007/978-3-642-16339-5_14 